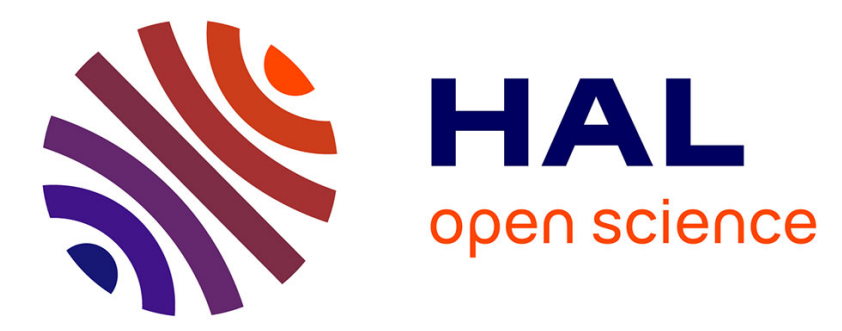

\title{
The sensitivity of surface mass loading displacement response to perturbations in the elastic structure of the crust and mantle
}

\author{
Hilary R. Martens, Luis Rivera, Mark Simons, Takeo Ito
}

\section{- To cite this version:}

Hilary R. Martens, Luis Rivera, Mark Simons, Takeo Ito. The sensitivity of surface mass loading displacement response to perturbations in the elastic structure of the crust and mantle. Journal of Geophysical Research: Solid Earth, 2016, 121 (5), pp.3911-3938. 10.1002/2015JB012456 . hal02497898

\section{HAL Id: hal-02497898 \\ https://hal.science/hal-02497898}

Submitted on 22 Oct 2021

HAL is a multi-disciplinary open access archive for the deposit and dissemination of scientific research documents, whether they are published or not. The documents may come from teaching and research institutions in France or abroad, or from public or private research centers.
L'archive ouverte pluridisciplinaire HAL, est destinée au dépôt et à la diffusion de documents scientifiques de niveau recherche, publiés ou non, émanant des établissements d'enseignement et de recherche français ou étrangers, des laboratoires publics ou privés.

$$
\text { Copyright }
$$




\section{Journal of Geophysical Research: Solid Earth}

\section{RESEARCH ARTICLE \\ 10.1002/2015JB012456 \\ Key Points: \\ The sensitivity of surface mass loading displacement response to perturbations in the elastic structure of the crust and mantle}

- We compute Love number and

Green's function sensitivity to shallow structural perturbations

-We compute the structural sensitivity of surface displacements induced by ocean tidal loading

- Load-induced displacements appear to be only weakly sensitive to density structure

Supporting Information:

- Supprting Information S1

- Supprting Information S2

- Data Set S1

- Data Set 52

- Data Set S3

- Data Set S4

- Data Set S5

- Data Set S6

- Data Set S7

- Data Set $\mathrm{S} 8$

- Data Set S9

- Data Set S10

- Data Set S11

- Data Set S12

- Data Set S13

- Data Set S14

- Data Set S15

- Data Set S16

- Data Set S17

- Data Set S18

- Data Set S19

- Data Set S20

- Data Set S21

- Data Set S22

- Data Set S23

- Data Set S24

Correspondence to:

H. R. Martens,

hmartens@caltech.edu

Citation:

Martens, H. R., L. Rivera, M. Simons, and T. Ito (2016), The sensitivity of surface mass loading displacement response to perturbations in the elastic structure of the crust and mantle, J. Geophys. Res. Solid Earth, 121, 3911-3938, doi:10.1002/2015JB012456.

Received 17 AUG 2015

Accepted 22 APR 2016

Accepted article online 28 APR 2016

Published online 19 MAY 2016

(2016. American Geophysical Union. All Rights Reserved.

\author{
Hilary R. Martens ${ }^{1}$, Luis Rivera ${ }^{2}$, Mark Simons ${ }^{1}$, and Takeo Ito $^{3}$ \\ ${ }^{1}$ Seismological Laboratory, Division of Geological and Planetary Sciences, California Institute of Technology, Pasadena, \\ California, USA, ${ }^{2}$ Institut de Physique du Globe de Strasbourg (UMR 7516), Université de Strasbourg/CNRS, Strasbourg, \\ France, ${ }^{3}$ Graduate School of Environmental Studies, Nagoya University, Nagoya, Japan
}

\section{Introduction}

Surface mass loading (SML) deforms the solid Earth in a manner controlled by the material properties of the interior. Examples of surface mass loads include oceans, lakes, rivers, reservoirs, the atmosphere, and seasonal precipitation. Since surface mass loads excite both elastic and gravitational responses in the solid Earth, we are motivated by the prospect of using observed SML-induced surface displacements, perhaps in combination with seismic observations, to probe the composition of the crust and mantle [e.g., Baker, 1980; Ito and Simons, 2011]. In addition to refining models of Earth's rheological structure, the geodetically inferred constraints on material properties could potentially shed light on mantle mechanics, such as the long-term stability of continental cratons [e.g., Jordan, 1978].

The concept of using SML-induced deformation to probe Earth's interior structure emerged several decades ago [e.g., Takeuchi, 1950; Longman, 1962, 1963; Farrell, 1972], yet early attempts to implement the theory using gravity, strain, and tilt measurements were limited in effectiveness due to insufficient spatial coverage of available observations, calibration uncertainties, and high sensitivities to local variations in material properties [e.g., Baker, 1980, 1984; Baker and Bos, 2003]. Space-based geodetic techniques, such as the Global Positioning System (GPS), do not suffer from the same sparsity or sensitivity constraints and may be used to discern centimeter level SML-induced surface displacements with submillimeter precision [e.g., Agnew, 2015; Penna et al., 2015; Martens et al., 2016].

One type of prominent surface mass loading comes from the periodic redistribution of ocean water by tidal forcing, known as ocean tidal loading (OTL). Although the theories that we discuss in this manuscript apply 
generally to the elastic displacement of the solid Earth in response to any surface mass load, we often refer to OTL-induced surface displacements as pertinent and illustrative examples.

Ito and Simons [2011] used residual OTL-induced surface displacements to invert for small deviations in the elastic moduli and density relative to the preliminary reference Earth model (PREM) [Dziewonski and Anderson, 1981] beneath the western United States. The study, however, inadvertently neglected the geocenter motion induced by the redistribution of surface mass when computing the forward model [e.g., Fu et al., 2012; Wu et al., 2012]. As a result, the displacement load Green's functions (LGFs) yielded OTL response predictions in a reference frame inconsistent with the corresponding GPS observations. Thus, the residual surface displacements, which the authors attributed to unmodeled Earth structure, primarily reflected the long-wavelength differences between the two reference frames. Furthermore, the study used just a single year of GPS data, assumed an errorless model for the solid Earth body tides (SEBTs), and disregarded contributions to the time series from minor tidal harmonics, which compounded the uncertainties in their derived Earth model [Yuan and Chao, 2012].

More recently, Yuan and Chao [2012] and Yuan et al. [2013] reported spatially coherent residuals between GPS-inferred and forward modeled OTL-induced surface displacements across a global distribution of sites located more than $150 \mathrm{~km}$ inland of the coast, where the influence of errors in the ocean tide models is significantly diminished. The regional-scale spatial coherency was interpreted to indicate possible deficiencies in the adopted SEBT model. Moreover, Penna et al. [2015] and Bos et al. [2015] found spatially coherent discrepancies between observed and predicted OTL-induced surface displacements across western Europe. Adjusting the value for the shear modulus in the asthenosphere by invoking frequency-dependent dissipation effects within the mantle improved the model fit to their observations. In addition, Martens et al. [2016] observed spatial coherency among residual $\mathrm{M}_{2}$ OTL-induced surface displacements across South America.

Given the inferred spatial coherencies in residual OTL-induced surface displacements, as well as the accuracy of modern Global Navigation Satellite System (GNSS) measurements [e.g., Penna et al., 2015] and of modern tide models [e.g., Stammer et al., 2014], the possibility to constrain Earth structure from observations of SML-induced deformation appears increasingly tractable. Prior to inversions for material properties, however, the sensitivity of the deformation response to perturbations in Earth structure must be investigated. Here we focus on the sensitivities of SML-induced displacements to systematic perturbations in the elasticity and density of the crust and mantle.

Previous studies that explored the level of structural sensitivity contained within load-generated response signals have focused primarily on comparisons between published LGFs for a few seismologically derived Earth models [e.g., Francis and Mazzega, 1990; Penna et al., 2008; Wang et al., 2012; Yuan et al., 2013], which cannot resolve the sensitivities to individual model parameters independently. In other words, comparisons of LGFs from different reference Earth models provide a general sense for the average magnitude and pattern of structural sensitivity but do not provide distinct information about the effects of layer thickness, perturbation depth, or elastic parameter. Vector differences between pairs of predicted OTL-induced surface displacements derived from various combinations of one-dimensional Earth models and modern ocean tide models are at the submillimeter level or less for most land-based locations [e.g., Penna et al., 2008; Wang et al., 2012; Yuan et al., 2013; Martens et al., 2016].

Isolating the influence of various factors, such as the particular elastic parameter and depth of the perturbation, can further elucidate details of Earth's elastic response to SML. In particular, Baker [1980] computed variations in tilt LGFs derived from individual perturbations to the two elastic moduli and density, albeit for only two separate layers in the crust and upper mantle. Other studies have also explored changes in the LGFs due to controlled differences in the material properties but focused solely on near-surface structure [e.g., Bos, 2010; Wang et al., 2012; Dill et al., 2015]. As expected, perturbations to crustal structure predominantly affect the high-degree load Love numbers and therefore the LGFs in the near field $\left(<1^{\circ}\right)$ [e.g., Baker, 1980; Francis and Mazzega, 1990].

For regional or global analyses of SML-generated deformation, however, mantle structure also has a significant influence on the deformation response [e.g., Ito and Simons, 2011]. Furthermore, in the case of OTL, coastal stations near the load, which are very sensitive to local crustal structure, are also highly susceptible to errors 
in the input tide model as well as to the method of coastline refinement adopted by the requisite convolution process and therefore may be of limited use in OTL-based geodetic tomography [Bos and Baker, 2005; Penna et al., 2008; Yuan et al., 2013].

Ito and Simons [2011] computed displacement LGF sensitivities numerically for perturbations to the two elastic moduli and density as a function of depth and distance to the load. They concluded that displacements excited by SML are most sensitive to elastic structural perturbations within a few hundred kilometers of the surface and also found a lack of trade-off between the kernels for density and the elastic moduli. The study did not, however, control the effects of layer thickness on response amplitude and also inadvertently contaminated the density kernel with extraneous perturbations to the elastic moduli. The contamination stemmed from parameterizing the input Earth model in terms of seismic velocities rather than the elastic moduli. In other words, density was perturbed with the $P$ wave $\left(V_{P}\right)$ and $S$ wave $\left(V_{S}\right)$ velocities held constant instead of the shear $(\mu)$ and bulk $(\kappa)$ moduli held constant, resulting in unintended perturbations to the elastic moduli with each density perturbation.

Adopting a more analytical approach, Okubo and Saito [1983] used calculus of variations to explore the sensitivities of potential, load, and shear Love numbers to independent perturbations of the two elastic moduli and density as a function of depth. Okubo and Endo [1986] expanded upon the theory of Okubo and Saito [1983] to address the special case of the degree 1 spherical harmonic. Further, Okubo [1988a] and Okubo [1988b] outlined a method to derive partial derivatives of the vertical and horizontal displacement LGFs from summations of the load Love number partial derivatives.

Here we quantify the sensitivities of Love numbers, displacement LGFs, and OTL-induced surface displacements to systematic perturbations in elastic and density structure through the crust and mantle at a variety of spatial scales. We begin by reviewing the numerical and quasi-analytical methods for computing partial derivatives of the Love numbers. Our results include a specific discussion of the partial derivatives for high-degree load Love numbers, which had not been included in previous studies. We then compute LGF sensitivity kernels numerically, revising and expanding upon the work of Ito and Simons [2011] by recomputing the density kernel as well as varying the layer thicknesses in a controlled manner. Finally, we perform a case study to quantify the sensitivity of OTL-induced surface displacements to systematic perturbations in elastic and density structure along two high-resolution $(\approx 1 \mathrm{~km}$ spacing) profiles across Iceland.

In summary, we compute sensitivity kernels for the Love numbers, displacement LGFs, and OTL-induced surface displacements as a function of (1) elastic model parameter, (2) displacement spatial component, (3) distance between the applied load and the measurement site, (4) depth of the perturbation, and (5) thickness of the perturbed layer. Our objective is to characterize the sensitivity of OTL-induced surface displacements to variations in elastic Earth structure. The techniques we develop here are directly applicable to future tomographic inversions using observations of SML-induced surface deformation. In particular, sensitivity kernels representing the effects of perturbed elastic material properties on the SML-induced surface displacements may be used to relate a model for Earth structure to the surface displacement observations in the linearized inverse problem.

\section{Methodology}

Love numbers are dimensionless parameters that characterize the elastic deformation of Earth to applied body forces and surface tractions [Love, 1911; Munk and MacDonald, 1960]. For example, we commonly represent the response of an elastic Earth to an external gravitational potential, $V$, by a set of three real-valued and dimensionless Love numbers: $h_{n}(r), k_{n}(r)$, and $I_{n}(r)$. The $I_{n}(r)$ parameter is alternatively referred to as the Shida number. Although the parameters exhibit a radial dependence, here we consider deformation observed only at Earth's surface and thus drop the $(r)$ notation.

The radial displacement, $u_{n}$, of Earth's surface in response to the application of an external gravitational potential of spherical harmonic degree, $n$, is given by [e.g., Agnew, 2015]

$$
u_{n}=h_{n} \frac{V_{n}}{g}
$$

where $g$ is the gravitational acceleration at Earth's surface, $\frac{v_{n}}{g}$ represents the equilibrium potential height, and $h_{n}$ scales the equilibrium height to a vertical displacement response commensurate with the density 
and elastic properties of Earth's interior. Gravitational self-attraction, generated by the redistributed mass, is accounted for in the response parameter $h_{n}$ [e.g., Munk and MacDonald, 1960]. Analogously, the Shida number $I_{n}$ is defined as the horizontal displacement response relative to the gradient of the equilibrium potential height. The parameter $k_{n}$ characterizes the change in the gravitational potential resulting from the redistribution of mass that occurs in response to the external potential field.

The Love and Shida numbers presented thus far describe the response of the elastic Earth to an external gravitational potential; thus, we refer to them as potential Love numbers. A second class of Love numbers, referred to as load Love numbers (LLNs), describes the elastic deformation of Earth in response to normal tractions, typically applied at Earth's surface [e.g., Munk and MacDonald, 1960; Longman, 1962; Saito, 1978]. External surface mass loads come from a variety of sources, including glaciers, lakes, the atmosphere, and oceans. The load Love numbers are distinguished from the potential Love numbers by a superscript prime: $h_{n}^{\prime}, l_{n}^{\prime}$ and $k_{n}^{\prime}$. A third class of Love numbers characterizes Earth's response to tangential tractions [e.g., Saito, 1978 ], distinguished from the other classes by superscript double primes $\left(h_{n}^{\prime \prime}, l_{n}^{\prime \prime}\right.$, and $\left.k_{n}^{\prime \prime}\right)$ and known as shear Love numbers. Only six of the nine Love numbers from the three sets (potential, load, and shear) are independent, and expressions exist to relate the Love numbers to one another [e.g., Molodenskiy, 1977; Saito, 1978; Lambeck, 1988].

To derive the various sets of Love numbers, we solve the equations of motion for spheroidal deformation of a self-gravitating, radially heterogeneous, spherically symmetric, nonrotating, elastic and isotropic (SNREI) Earth [e.g., Alterman et al., 1959; Longman, 1962; Takeuchi and Saito, 1972]. The equations of motion are given by

$$
\begin{aligned}
& \dot{y}_{1}=\frac{-2 \lambda}{A} \frac{y_{1}}{r}+\frac{y_{2}}{A}+\frac{\lambda C}{A} \frac{y_{3}}{r}, \\
& \dot{y}_{2}=\left[-\omega^{2} \rho r^{2}-4 \rho g r+\frac{4 \mu B}{A}\right] \frac{y_{1}}{r^{2}}-\frac{4 \mu}{A} \frac{y_{2}}{r}+\left[C \rho g r-\frac{2 \mu B C}{A}\right] \frac{y_{3}}{r^{2}}+C \frac{y_{4}}{r}-\rho y_{6}, \\
& \dot{y}_{3}=-\frac{y_{1}}{r}+\frac{y_{3}}{r}+\frac{y_{4}}{\mu}, \\
& \dot{y}_{4}=\left[g \rho r-\frac{2 \mu B}{A}\right] \frac{y_{1}}{r^{2}}-\frac{\lambda}{A} \frac{y_{2}}{r}+\left[-\omega^{2} \rho r^{2}+\frac{2 \mu}{A}\left[\lambda\left(2 n^{2}+2 n-1\right)+2 \mu\left(n^{2}+n-1\right)\right]\right] \frac{y_{3}}{r^{2}}-\frac{3 y_{4}}{r}-\rho \frac{y_{5}}{r}, \\
& \dot{y}_{5}=4 \pi G \rho y_{1}+y_{6}, \\
& \dot{y}_{6}=-4 \pi G \rho C \frac{y_{3}}{r}+C \frac{y_{5}}{r^{2}}-\frac{2 y_{6}}{r},
\end{aligned}
$$

where $A=\lambda+2 \mu, B=3 \lambda+2 \mu, C=n(n+1), \lambda$ and $\mu$ are Lamé parameters, $\rho$ is density, $g$ is gravity, $G$ is the universal gravitational constant, and $\omega$ is the forcing frequency. The variables $y_{1}$ and $y_{3}$ characterize the radial and tangential displacements, respectively; $y_{2}$ and $y_{4}$ characterize the radial and tangential stress, respectively; $y_{5}$ characterizes the gravitational potential; and the equation for $\dot{y}_{5}$ defines $y_{6}$. Dots represent differentiation with respect to $r$. As an aside, we note that Takeuchi and Saito [1972] adopt a different convention for the definition of $y_{6}$, which requires a slight adjustment to the surface boundary conditions.

Only three of the six linearly independent solutions to the equations of motion (equation (2)) are bounded at the origin. We compute the three sets of starting solutions using analytical formulae for a homogeneous sphere [Takeuchi and Saito, 1972]. We then propagate the three solution sets through a radially heterogeneous Earth model to the surface for each spherical harmonic degree. Appropriate boundary conditions are applied across each internal interface, including solid-fluid boundaries [Takeuchi and Saito, 1972], as well as at the surface. Our internally developed software package (LoadDef) integrates the equations of motion using a Runge-Kutta algorithm and adaptive step sizing. Beyond spherical harmonic degree 20 , we begin integration within the mantle for reasons of numerical stability. We retain the inertial factors in the equations of motion and set the forcing frequency equivalent to the $\mathrm{M}_{2}$ tidal harmonic.

Although here we focus primarily on LLNs derived for mass loading boundary conditions at the surface [e.g., Longman, 1962, 1963; Melchior, 1983; Guo et al., 2004], additional Love numbers, including potential and shear, may be computed by adopting alternative surface boundary conditions, several of which are listed in Table 1.

To compute the displacement LGFs, which represent the response to a delta function unit normal force at Earth's surface, we combine the LLNs in spherical harmonic expansions [Farrell, 1972]. The amplitudes of the 
Table 1. Summary of Surface Boundary Conditions and Love Number Definitions for the Cases of (A) the Presence of an External Gravitational Potential, (B) Surface Mass Loading, (C) Surface Shear Forcing, and (D) Surface Stress Conditions [Longman, 1962, 1963; Wiggins, 1968; Farrell, 1972; Lanzano, 1982; Melchior, 1983; Okubo and Saito, 1983; Okubo and Endo, 1986; Guo et al., 2004] ${ }^{\mathrm{a}}$

\begin{tabular}{|c|c|c|c|c|}
\hline & $\begin{array}{c}\quad(\mathrm{A}) \\
\text { External } \\
\text { Potential }\end{array}$ & $\begin{array}{c}\text { (B) } \\
\text { Surface } \\
\text { Mass Loading }\end{array}$ & $\begin{array}{c}(\mathrm{C}) \\
\text { Surface } \\
\text { Shear Forcing }\end{array}$ & $\begin{array}{c}\text { (D) } \\
\text { Surface } \\
\text { Stress }(n=1) \\
\end{array}$ \\
\hline \multicolumn{5}{|c|}{ Surface Boundary Conditions } \\
\hline$y_{2}$ & 0 & $-g_{S}^{2} \frac{2 n+1}{4 \pi G}$ & 0 & $-\frac{3 g_{S}^{2}}{4 \pi G}$ \\
\hline$y_{4}$ & 0 & 0 & $\frac{(2 n+1) g_{S}^{2}}{4 \pi G n(n+1)}$ & $\frac{3 g_{S}^{2}}{8 \pi G}$ \\
\hline$y_{6}+\frac{n+1}{a} y_{5}$ & $(2 n+1) g_{S}$ & $(2 n+1) g_{S}$ & 0 & 0 \\
\hline \multicolumn{5}{|c|}{ Love Numbers } \\
\hline$y_{1} / a$ & $h_{n}$ & $h_{n}^{\prime}$ & $h_{n}^{\prime \prime}$ & $h_{1}^{\prime \prime \prime}$ \\
\hline$y_{3} / a$ & $I_{n}$ & $l_{n}^{\prime}$ & $I_{n}^{\prime \prime}$ & $l_{1}^{\prime \prime \prime}$ \\
\hline$y_{5} /\left(a g_{S}\right)$ & $\left(k_{n}+1\right)$ & $\left(k_{n}^{\prime}+1\right)$ & $k_{n}^{\prime \prime}$ & $k_{1}^{\prime \prime \prime}$ \\
\hline
\end{tabular}

${ }^{a}$ The surface stress solution satisfies the consistency relation and thus provides an important linearly independent solution for the evaluation of degree 1 modes [Okubo and Endo, 1986]. Note that our definitions for the Love numbers and surface boundary conditions differ from Okubo and Saito [1983] by a factor of $a g_{S}$, where $a$ is Earth's radius and $g_{S}$ is the gravitational acceleration at Earth's surface.

vertical and horizontal displacement LGFs, per unit of load mass, are given by

$$
u(\theta)=\frac{a}{m_{E}} \sum_{n=0}^{\infty} h_{n}^{\prime} P_{n}(\cos \theta)
$$

and

$$
v(\theta)=\frac{a}{m_{E}} \sum_{n=1}^{\infty} I_{n}^{\prime} \frac{\partial P_{n}(\cos \theta)}{\partial \theta}
$$

where $a$ is the Earth radius, $m_{E}$ is the Earth mass, $n$ represents the spherical harmonic degree, $P_{n}$ represents the Legendre polynomial of spherical harmonic degree $n, \theta$ represents the angular distance between a measurement site and the load point, $h_{n}^{\prime}$ is the vertical displacement load Love number, and $l_{n}^{\prime}$ is the horizontal displacement load Love number.

To facilitate convergence of the LGFs, we compute asymptotic expressions of the LLNs [Guo et al., 2004] and apply Kummer's series transformation to equations (3) and (4) [e.g., Abramowitz and Stegun, 1964; Farrell, 1972; $\mathrm{Na}$ and Baek, 2011]. In practice, the LLN computations are carried out to spherical harmonic degree 10,000, beyond which the LLNs are assumed to be equivalent to the asymptotic values [Farrell, 1972; Guo et al., 2004]. The asymptotic expressions are generally accurate to at least $0.01 \%$ and often accurate to within $0.0001 \%$ of the true values at $n=10,000$.

In summary, the displacement LGFs are computed using the formulae

$$
u(\theta) \approx \frac{a}{m_{E}} h_{\infty}^{*} \sum_{n=1}^{\infty} P_{n}(\cos \theta)+\frac{a}{m_{E}} h_{\infty}^{* *} \sum_{n=1}^{\infty} \frac{1}{n} P_{n}(\cos \theta)+\frac{a}{m_{E}} h_{0}^{\prime}+\frac{a}{m_{E}} \sum_{n=1}^{N=10,000}\left(h_{n}^{\prime}-\left(h_{\infty}^{*}+\frac{1}{n} h_{\infty}^{* *}\right)\right) P_{n}(\cos \theta)
$$

for the vertical displacement response and

$$
v(\theta) \approx \frac{a}{m_{E}} l_{\infty}^{*} \sum_{n=1}^{\infty} \frac{1}{n} \frac{\partial P_{n}(\cos \theta)}{\partial \theta}+\frac{a}{m_{E}} l_{\infty}^{* *} \sum_{n=1}^{\infty} \frac{1}{n^{2}} \frac{\partial P_{n}(\cos \theta)}{\partial \theta}+\frac{a}{m_{E}} \sum_{n=1}^{N=10,000}\left(n l_{n}^{\prime}-\left(l_{\infty}^{*}+\frac{1}{n} l_{\infty}^{* *}\right)\right) \frac{1}{n} \frac{\partial P_{n}(\cos \theta)}{\partial \theta}
$$

for the horizontal displacement response, where $h_{\infty}^{*}$ and $h_{\infty}^{* *}$ represent the first- and second-order coefficients of the asymptotic expansions, respectively, for the vertical displacement LLN (i.e., $\lim _{n \rightarrow \infty} h_{n}^{\prime} \approx h_{\infty}^{*}+\frac{1}{n} h_{\infty}^{* *}$ ) and $l_{\infty}^{*}$ and $l_{\infty}^{* *}$ represent the first- and second-order coefficients of the asymptotic expansions, respectively, 
for the horizontal displacement LLN (i.e., $\lim _{n \rightarrow \infty} n l_{n}^{\prime} \approx l_{\infty}^{*}+\frac{1}{n} *_{\infty}^{* *}$ ) [Guo et al., 2004]. Because the analytical expression for the second-order Legendre sum (second term in equation (6)) becomes undefined for $\theta=180^{\circ}$, the displacement LGFs at that angular distance are computed by extrapolation of neighboring values with a resolution of 1 in 1000 [Guo et al., 2004].

Since estimates of site positions derived from analysis of GPS observations are usually referred to the center of mass of the entire Earth system (CM) [e.g., Wu et al., 2012; Agnew, 2015], we convert the LGFs, computed initially in a solid Earth centered (CE) reference frame, to the CM reference frame by making the appropriate modifications to the degree 1 LLNs [Blewitt, 2003]:

$$
\begin{aligned}
{\left[h_{1}^{\prime}\right]_{\mathrm{CM}} } & =\left[h_{1}^{\prime}\right]_{\mathrm{CE}}-1 \\
{\left[l_{1}^{\prime}\right]_{\mathrm{CM}} } & =\left[l_{1}^{\prime}\right]_{\mathrm{CE}}-1 \\
{\left[1+k_{1}^{\prime}\right]_{\mathrm{CM}} } & =\left[1+k_{1}^{\prime}\right]_{\mathrm{CE}}-1 .
\end{aligned}
$$

To predict SML-induced surface displacements for a load of finite size, we convolve the displacement LGFs with a model for the surface mass load [e.g., Farrell, 1973; Baker, 1984; Agnew, 2015]. The equation for the predicted displacement response is given by

$$
U\left(r, S, \rho_{z}, Z\right)=\int_{\Omega^{\prime}} G\left(\left|r-r^{\prime}\right|, S\right) \rho_{z}\left(r^{\prime}\right) Z\left(r^{\prime}\right) \mathrm{d} \Omega^{\prime},
$$

where $U$ is the SML-induced surface displacement at observation point $r, \rho_{z}$ is the mass density of the load at the load point $r^{\prime}, G$ is the LGF representing the displacement response of a radially symmetric Earth to a $1 \mathrm{~kg}$ point load, and $Z$ represents the height of the load at point $r^{\prime}$. The integral is taken over the entire surface of the Earth, $\Omega^{\prime}$. Note that the LGF depends on distance to the load as well as Earth structure, $S$, which varies with radius inside the SNREI Earth (e.g., PREM). Thus, the predicted response, $U$, depends on the position of the measurement site relative to the applied load as well as on Earth structure and the particular load model.

For the special case of OTL, $\rho_{z}$ is the density of seawater, $Z$ is complex valued to represent both the amplitude and phase of the tide height, and the integral is evaluated only over the surface area of the oceans. Since ocean tidal loads are complex valued, $U$ also becomes complex valued, returning both amplitude and phase components for the predicted OTL-induced surface displacements. The frequency of the response is equivalent to the frequency of the tidal harmonic.

To complete the workflow, we compute the entire forward model, from integration of the equations of motion to the convolution of the LGFs with a load model, within our LoadDef software. Our evaluation of equation (8), however, is closely modeled after the SPOTL package [Agnew, 1997, 2012]. In particular, LoadDef adopts a station-centered template grid, which simplifies the ability to refine the integration grid around a station, include multiple loading models, and compute specific LGFs at the grid nodes [Goad, 1980; Agnew, 1997, 2012].

Since the mass loads that we consider are confined to Earth's surface, equation (8) may be expanded as [e.g., Harrison, 1985; Agnew, 1997]

$$
U\left(r, S, \rho_{z}, Z\right)=\int_{0}^{2 \pi} \int_{0}^{\pi} G(\theta, S) \rho_{z}(\theta, \alpha) Z(\theta, \alpha) T(\alpha) a^{2} \sin \theta \mathrm{d} \theta \mathrm{d} \alpha,
$$

where $\theta$ is the angular distance between the observer at $r$ and a particular load point; $T(\alpha)$ is a trigonometric factor used to decompose the predicted horizontal displacements into separate vector components (for the vertical displacements, $T(\alpha)=1$ ); $\alpha$ is the azimuth of a particular load point relative to the observer, measured clockwise from north; and $a$ is Earth's radius. Note that for a station-centered template grid, the station is considered to be located at the pole of a spherical coordinate system, where $\theta$ is the polar angle and $\alpha$ is the azimuthal angle.

We evaluate equation (9) using numerical integration methods. Specifically, we discretize the surface integral into finite-sized cells and evaluate the integrand at the midpoint of each cell. Since the LGFs vary most rapidly in the near field, we increase the spatial resolution of the grid substantially in the immediate vicinity of the station (measurement site) by reducing the increment in the polar angle, $\Delta \theta$. The increment in the polar angle 
may be greater in the far field since changes in the LGF as a function of $\theta$, as well as the absolute value of the LGF, diminish dramatically at large $\theta$. We therefore generate an integration grid with tapered resolution as a function of distance to the station: $\Delta \theta=0.001^{\circ}$, or about $100 \mathrm{~m}$, within $\theta=1^{\circ} ; \Delta \theta=0.01^{\circ}$ from $\theta=1-10^{\circ}$; $\Delta \theta=0.1^{\circ}$ from $\theta=10-90^{\circ}$; and $\Delta \theta=1.0^{\circ}$ beyond $\theta=90^{\circ}$. For each $\theta$ in the integration grid, we set the increment in the azimuthal angle, $\Delta \alpha$, to $0.1^{\circ}$. The integration over finite grid cells alleviates the problem of the singularity in the LGFs at $\theta=0$, which we also mitigate prior to integration using suitable normalization factors [Agnew, 1997, 2012].

Since the integration mesh is irregular, standard methods for estimating the quadrature accuracy cannot be directly applied [e.g., Press et al., 2007, chap. 4]. Given the numerical integration technique described above, an analytical estimation of the quadrature error would require an evaluation of the variation in the second derivative of the integrand from equation (9) over each cell. Instead, we compare predicted displacements derived from grids of different resolution to place bounds on the uncertainty in the discrete convolution. Using this strategy, we estimate that the bounds on the absolute error in the predicted displacements, $U$, are on the order of $0.01-0.1 \mathrm{~mm}$ for $\mathrm{M}_{2}$ OTL. Furthermore, we are primarily concerned with the vector differences, $\left|U_{1}-U_{2}\right|$, between pairs of predicted OTL-induced surface displacements derived from different Earth models. Since the derivatives of the integrand in equation (9) do not vary much for small perturbations to Earth structure, the bounds on the quadrature error may be reduced to about $0.1 \mu \mathrm{m}$ when considering the vector differences between pairs of predicted displacements.

In addition to quadrature errors, the discrete tide models can be imprecise near coastal boundaries. Thus, we refine the ocean tide models around the coastlines by first extrapolating the complex-valued tide heights inland by one grid cell, then interpolating the tide model onto the integration grid, and finally, applying a land-sea mask based on ETOPO1 [Amante and Eakins, 2009] in the far field and Global Self-consistent, Hierarchical, High-resolution Shoreline database (GSHHS) [Wessel and Smith, 1996] within $1.5^{\circ}$ of the measurement site. ETOPO1 provides global topographic and bathymetric relief information at 1 arc min resolution. GSHHS provides global shoreline information, which we adopt at full resolution. Around the Antarctic, we allow the ocean model grid to define the coastline since ETOPO1 registers floating ice shelves as landmasses, but the tides remain active in those regions.

Although the tide models, method of coastline refinement, and adopted values for seawater density have been shown to generate erroneous OTL-induced displacement predictions at the level of $\approx 1-5 \%$ [e.g., Bos and Baker, 2005; Penna et al., 2008], here we only consider the differential displacement response. Thus, our results are not sensitive to the usual, and often dominant, sources of prediction error. In other words, we focus on perturbing the structural properties of the solid Earth while keeping the load model, load density, and convolution procedure consistent throughout each comparison.

\section{Results}

\subsection{Love Number Sensitivities}

We first explore the sensitivity of Love numbers to perturbations in the elastic moduli, $\mu$ and $\kappa$, and density, $\rho$. The sensitivity kernels, $K$, are computed as

$$
K_{p, H}^{j}=\frac{H\left(\mathbf{m}+\Delta \mathbf{m}_{p}^{j}\right)-H(\mathbf{m})}{\Delta \mathbf{m}_{p}^{j}}=\frac{\Delta H}{\Delta \mathbf{m}_{p}^{j}},
$$

where $j$ corresponds to a particular perturbed layer, $p$ corresponds to the model parameter being perturbed $(\mu, \kappa$, or $\rho)$, and $H$ corresponds to a particular Love number, which depends on Earth structure, $\mathbf{m}$. The perturbation to structure, $\Delta \mathbf{m}_{p}^{j}$, involves a perturbation to the $p$ model parameter in layer $j$; all other model parameters remain unperturbed.

The sensitivity kernels may be computed both numerically [Ito and Simons, 2011] and quasi-analytically [Okubo and Saito, 1983; Okubo and Endo, 1986; Okubo, 1988a]. For the numerical computation, we derive the Love numbers using two Earth models, the reference model and the perturbed model, and compute the finite differences explicitly. For the quasi-analytical computation, we follow the procedure of Okubo and Saito [1983] to derive the partial derivatives of the Love numbers, supplemented by the theory of Okubo and Endo [1986] for the special case of spherical harmonic degree $n=1$. We consider only perturbations to the solid mantle 

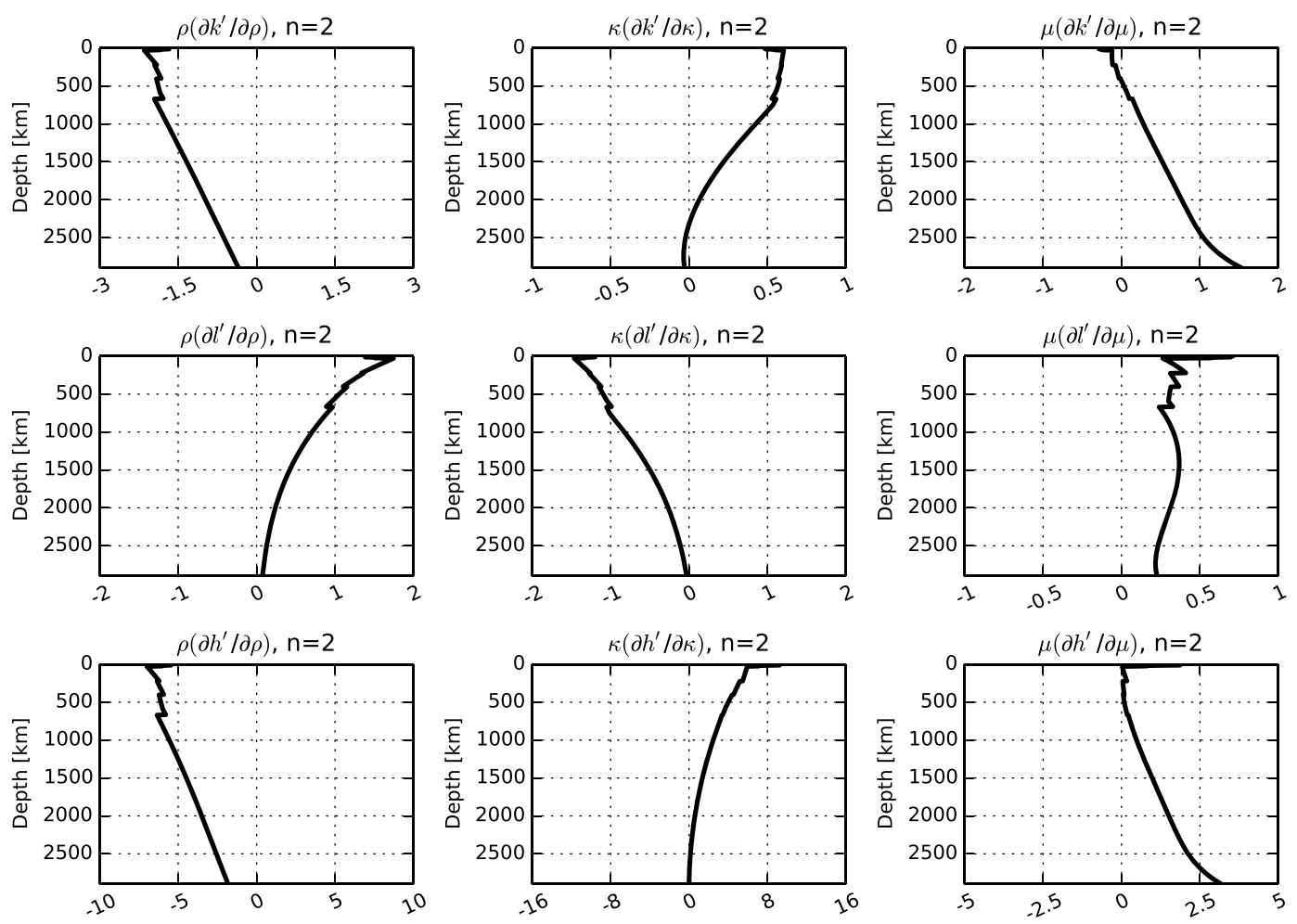

Figure 1. Partial derivatives of degree 2 load Love numbers with respect to the shear modulus, $\mu$, the bulk modulus, $\kappa$, and density, $\rho$, for Earth model PREM [Dziewonski and Anderson, 1981]. The partials have been multiplied by the depth profile of each elastic parameter, making them dimensionless. The horizontal axes are in units of $10^{-4} \mathrm{~km}^{-1}$. The figure may be compared with Figure $1 \mathrm{~b}$ in Okubo and Saito [1983], which was computed for Earth model 1066A.

and disregard the core regions. Unlike the SEBTs, the deformational influence of OTL is concentrated primarily within the upper mantle and crust, thereby justifying our neglect of perturbations to core structure [e.g., Ito and Simons, 2011; Bos et al., 2015].

The quasi-analytical technique, which is rooted in variational calculus, employs the same approach used to derive partial derivatives of surface wave phase velocities in seismology [e.g., Jeffreys, 1961; Takeuchi and Saito, 1972]. In particular, the method takes advantage of Rayleigh's principle to estimate variations in the LLNs due to small perturbations in the elastic and density structure, without relying on numerical differentiation [Okubo and Saito, 1983].

Integrated combinations of the partial derivatives yield the predicted variations in the Love numbers [Takeuchi and Saito, 1972; Okubo and Saito, 1983]. Formally, the change in a Love number due to elastic structural perturbations is given by

$$
\delta H_{n}=\int_{\mathrm{CMB}}^{a}\left\{\left[\frac{\partial H_{n}}{\partial \rho}(r)\right]_{\kappa \mu} \delta \rho(r)+\left[\frac{\partial H_{n}}{\partial \kappa}(r)\right]_{\mu \rho} \delta \kappa(r)+\left[\frac{\partial H_{n}}{\partial \mu}(r)\right]_{\kappa \rho} \delta \mu(r)\right\} \mathrm{d} r
$$

when we use $(\rho, \kappa, \mu)$ as independent parameters. In equation (11), $H_{n}$ represents a particular Love number (e.g., $h_{n}^{\prime}, l_{n}^{\prime}, k_{n}^{\prime}, h_{n}$, or $h_{n}^{\prime \prime}$ ), CMB represents the core-mantle boundary, $a$ is the Earth radius, and the partial derivatives are defined per unit thickness of the perturbed layer.

Figure 1 shows partial derivatives of degree 2 LLNs based on the crust and mantle structure of PREM. The partial derivatives are computed with respect to the bulk modulus, $\kappa$, shear modulus, $\mu$, and density, $\rho$. Partial derivatives of the potential, load, and shear Love numbers derived from PREM for additional spherical harmonic degrees as well as from reference Earth model 1066A [Gilbert and Dziewonski, 1975] for spherical harmonic degree 2 [cf. Okubo and Saito, 1983, Figure 1] are provided in the supporting information (Figures S1-S17). As noted by Okubo and Saito [1983], the partial derivatives of $h_{2}^{\prime}$ with respect to $\kappa$ and of $l_{2}^{\prime \prime}$ with respect to $\mu$ are largest in the crust due to the strong influence of compressibility and rigidity on normal and tangential tractions, respectively, applied at the surface. 
Table 2. Comparison of Degree 2 Load Love Number Partial Derivatives for a Homogeneous Sphere, Computed Quasi-Analytically Using Calculus of Variations and Numerically Using Finite Differences ${ }^{a}$

\begin{tabular}{lccccccc} 
& Quasi- & \multicolumn{7}{c}{ Magnitude of Linear Perturbation } \\
& $\begin{array}{c}\text { Analytical } \\
\text { Solution }\end{array}$ & $+0.1 \%$ & $+0.5 \%$ & $+1.0 \%$ & $+5.0 \%$ & $+10.0 \%$ & $+20.0 \%$ \\
\cline { 2 - 7 } & 0.5891 & 1.0008 & 1.0040 & 1.0079 & 1.0396 & 1.0792 & 1.1580 \\
\hline$\partial h_{2}^{\prime} / \partial \mu_{\text {norm }}$ & 0.1292 & 1.0008 & 1.0040 & 1.0079 & 1.0396 & 1.0793 & 1.1593 \\
$\partial l_{2}^{\prime} / \partial \mu_{\text {norm }}$ & 0.0793 & 1.079 & 1.0810 & 1.1620 \\
$\partial k_{2}^{\prime} / \partial \mu_{\text {norm }}$ & 0.3374 & 1.0008 & 1.0041 & 1.0081 & 1.0405 & 1.0810 \\
$\partial h_{2}^{\prime} / \partial \kappa_{\text {norm }}$ & 0.2409 & 1.0011 & 1.0056 & 1.0113 & 1.0563 & 1.1126 & 1.2251 \\
$\partial l_{2}^{\prime} / \partial \kappa_{\text {norm }}$ & -0.0851 & 1.0011 & 1.0056 & 1.0113 & 1.0563 & 1.1126 & 1.2252 \\
$\partial k_{2}^{\prime} / \partial \kappa_{\text {norm }}$ & 0.0194 & 1.0011 & 1.0057 & 1.0113 & 1.0566 & 1.1132 & 1.2263 \\
$\partial h_{2}^{\prime} / \partial \rho_{\text {norm }}$ & -1.3124 & 0.9980 & 0.9957 & 0.9929 & 0.9703 & 0.9423 & 0.8865 \\
$\partial l_{2}^{\prime} / \partial \rho_{\text {norm }}$ & 0.1050 & 0.9994 & 0.9891 & 0.9765 & 0.8821 & 0.7793 & 0.6129 \\
$\partial k_{2}^{\prime} / \partial \rho_{\text {norm }}$ & -0.4139 & 0.9974 & 0.9962 & 0.9947 & 0.9829 & 0.9687 & 0.9408 \\
\hline
\end{tabular}

${ }^{a}$ The model parameters considered here are the shear modulus, $\mu$, bulk modulus, $\kappa$, and density, $\rho$. The original (unperturbed) homogeneous sphere has properties of $V_{P}=10,000 \mathrm{~m} \mathrm{~s}^{-1}, V_{S}=5000 \mathrm{~m} \mathrm{~s}^{-1}$, and $\rho=5000 \mathrm{~kg} \mathrm{~m}^{-3}$. The perturbations are computed as a linear percentage of the original model and applied to the entire sphere. All parameters were normalized: $\mu$ and $\kappa$ by a factor $\left[\bar{\rho} \times a^{2} \times \bar{t}^{-2}\right]$ and $\rho$ by a factor $\bar{\rho}$, where $a$ is Earth's radius $(6,371,000 \mathrm{~m}), \bar{\rho}$ is Earth's approximate mean density $\left(5500 \mathrm{~kg} / \mathrm{m}^{3}\right)$, and $\bar{t}=\frac{1}{\sqrt{\bar{\rho} \pi G}}$. The Love numbers are, by definition, nondimensional. Note that the quasi-analytical partial derivatives are derived independent of a specified perturbation to the elastic parameters (i.e., they do not require explicit numerical differentiation).
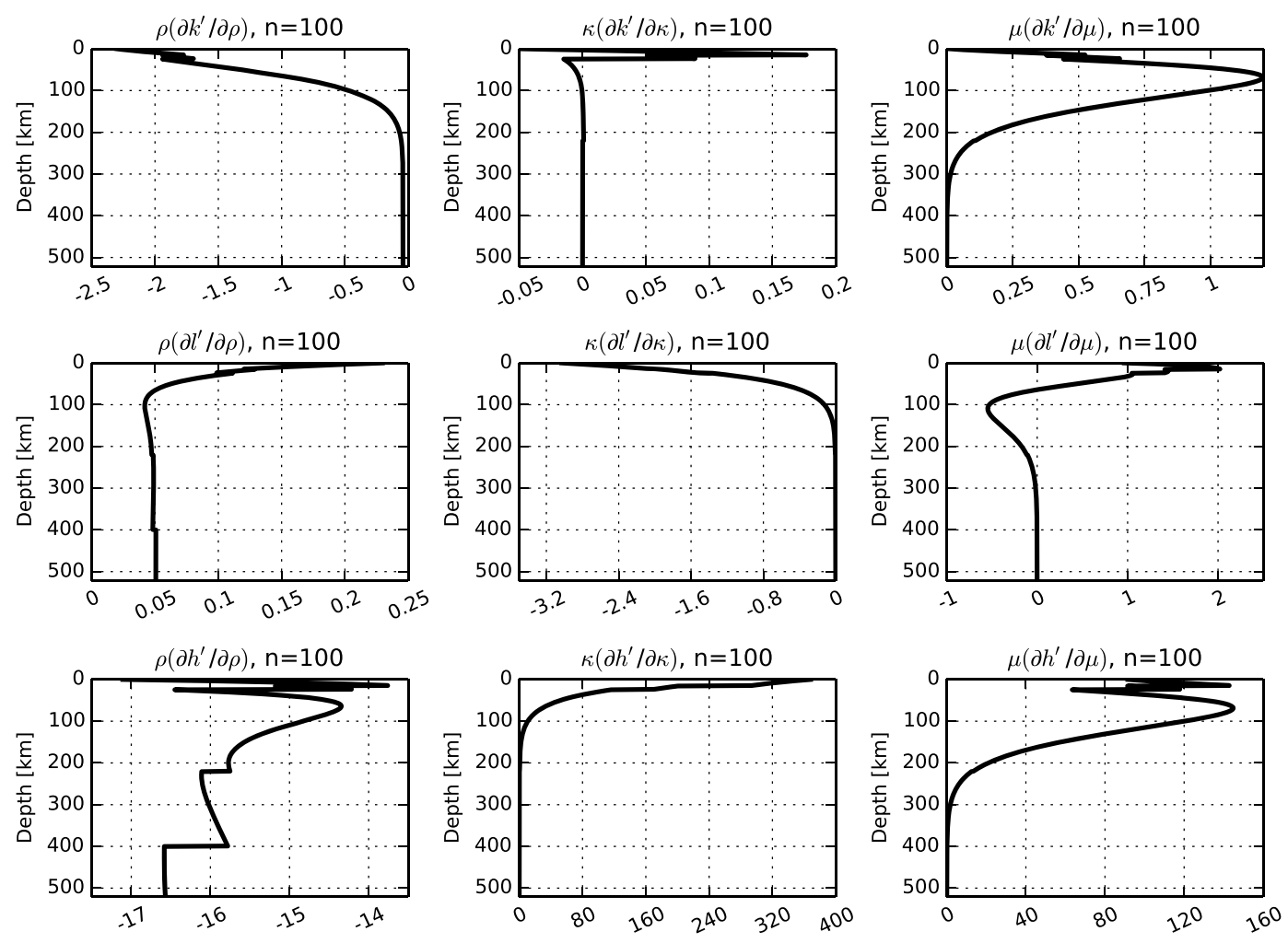

Figure 2. Same as Figure 1 but for spherical harmonic degree $n=100$. Note that the sensitivity drops off rapidly beneath about $300 \mathrm{~km}$ depth from the surface. Perturbations to the density structure at deeper depths continue to affect the load Love numbers due to the associated change in mass. 

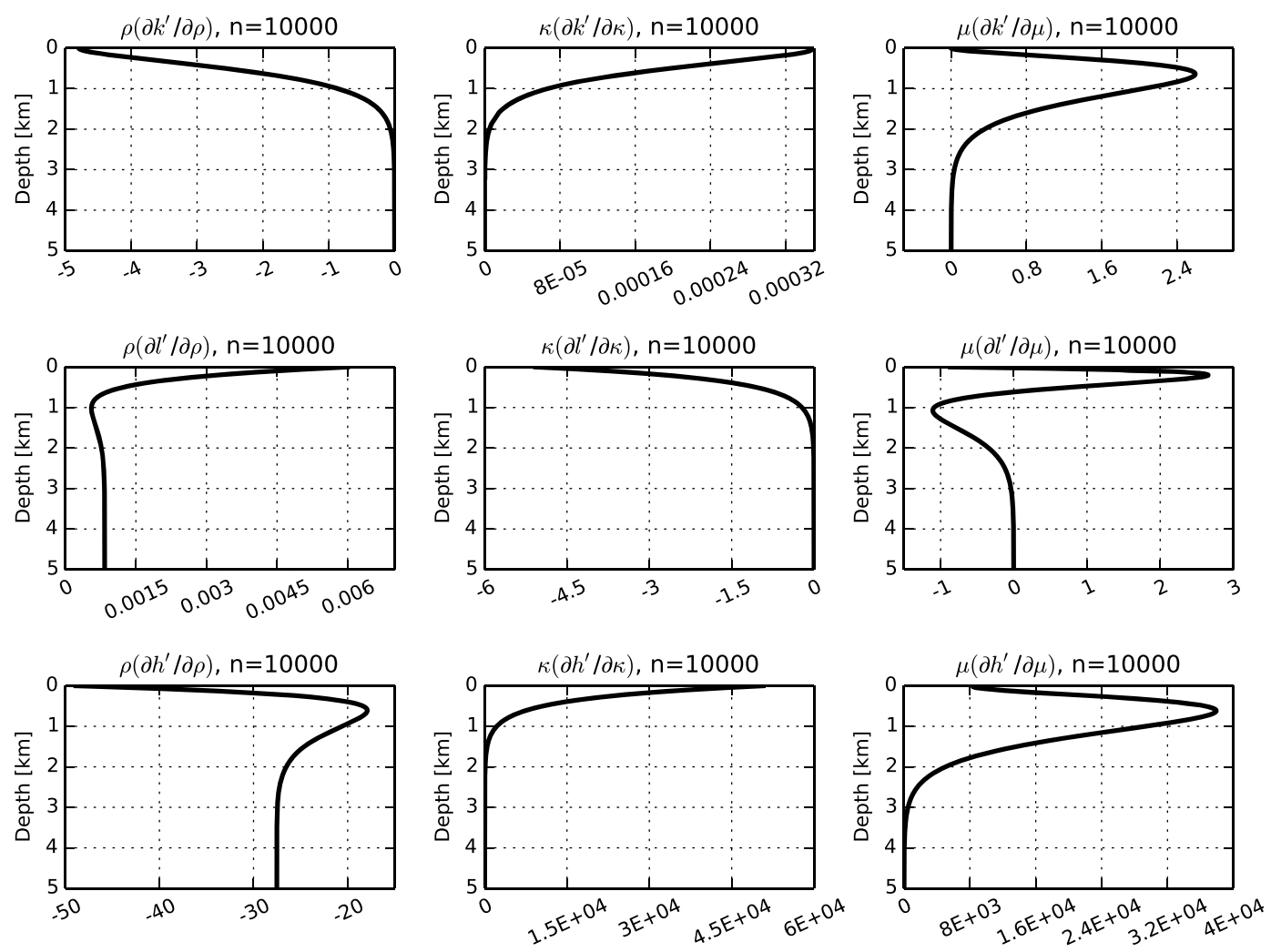

Figure 3. Same as Figure 1 but for spherical harmonic degree $n=10,000$. Note that the sensitivity drops off rapidly beneath about $3 \mathrm{~km}$ depth from the surface. Furthermore, we note that the magnitude range of the partial derivatives for the vertical displacement load Love number, $h^{\prime}$, at $n=10,000$ is significantly larger than for the lower spherical harmonic degrees (cf. Figures 1 and 2), indicating that the sensitivities are heightened for the higher degrees but also limited to the very near surface.

The quasi-analytical computation, although more complicated to implement than the numerical approach, reduces overall processing time since the Love numbers need only be computed once for a given Earth model. The development of the quasi-analytical approach outlined by Okubo and Saito [1983], however, involves a Taylor series expansion truncated to first order. To investigate the consequences of a first-order truncation to the series on the computed sensitivities, we compare the quasi-analytically and numerically derived partial derivatives of degree 2 LLNs for a homogeneous sphere model in Table 2. Specifically, we compare ratios of the quasi-analytical partial derivatives to the numerical partial derivatives for a variety of model parameter perturbations.

Note that we compute the quasi-analytical partial derivatives independent of any specified perturbation to a model parameter, since the quasi-analytical approach avoids explicit numerical differentiation [Jeffreys, 1961]. For linear perturbations to the model parameters of $1 \%$ or less, the quasi-analytically and numerically derived LLN partial derivatives of degree 2 differ by less than about $1 \%$. On the contrary, for $10 \%$ perturbations to the model parameters, the two methods of computation generate LLN partial derivatives that differ on the order of $10 \%$.

Partial derivatives of the LLNs at higher degrees are shown in Figures 2 and 3 as well as in the supporting information. As the spherical harmonic degree increases, the "skin depth" of the sensitivity decreases. For $n=100$ (Figure 2), the sensitivity of the LLNs to perturbations in the elastic moduli effectively drops to zero $\left(<10^{-4}\right.$ of the peak sensitivity) below about $300-400 \mathrm{~km}$ depth; for $n=10,000$ (Figure 3 ), the sensitivity effectively drops to zero below about $3-4 \mathrm{~km}$ depth. The observations are not surprising, since the load-induced displacements are proportional to $(r / a)^{n}$, where $r$ is a particular radius within the Earth, $a$ is Earth's radius, and $n$ is the spherical harmonic degree [e.g., Farrell, 1972]. Note as well the significant increase in peak sensitivity at higher degrees. The most striking variations in peak sensitivity occur for the partial derivatives of the vertical displacement LLN, $h^{\prime}$, with respect to perturbations in the two elastic moduli (cf. Figures 1-3). 

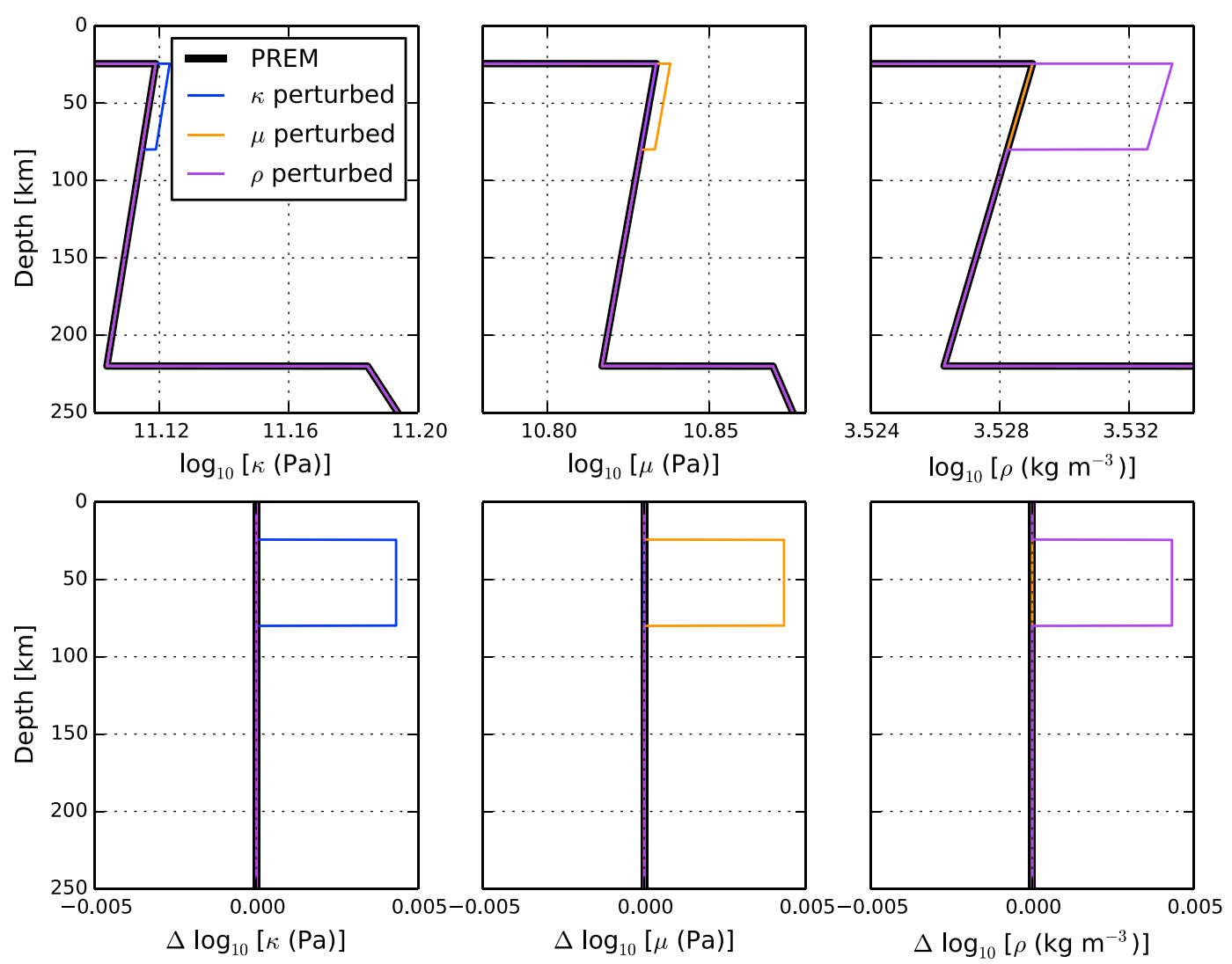

Figure 4. Three Earth models derived from perturbations applied to PREM in the upper mantle from 80 to $24.4 \mathrm{~km}$ depth. (top row) Profiles of the bulk modulus (left), shear modulus (middle), and density (right) for each Earth model as a function of depth. (bottom row) The differences in the bulk modulus (left), shear modulus (middle), and density (right) profiles between each Earth model and the unperturbed PREM. All profiles are depicted in common log space. To generate the three models, we perturbed the bulk modulus, shear modulus, and density separately by a factor of $1 \%$ in linear space or $\log _{10}(1.01)=0.0043$ in common log space. The perturbed Earth models are used to explore the sensitivities of load Green's functions (Figure 5) and OTL-induced surface displacements (Figure 14) to small perturbations in Earth structure.

The characteristic profiles of the LLN partial derivatives allow us to both visualize and quantify the sensitivity of the Love numbers to perturbations applied to individual structural parameters at each depth and for each spherical harmonic degree.

The work done to compute the LLN partial derivatives may be extended without much additional effort to compute partial derivatives of the potential and shear Love numbers as well [Okubo and Saito, 1983]. We show partial derivatives of the potential and shear Love numbers, derived from PREM for spherical harmonic degrees $2-4$, in the supporting information. Table $\mathrm{S} 1$ lists vertical and horizontal displacement potential Love numbers, $h$ and $l$, for several seismologically derived Earth models.

\subsection{Load Green's Function Sensitivities}

We now consider the influence of small (1\%) perturbations in the elastic and density structure on the displacement LGFs (equations (5) and (6)). To illustrate our methodology, we first perturb the bulk modulus, shear modulus, and density separately for a single layer of PREM within the upper mantle (80-24.4 km depth). We adopt an isotropic and oceanless version of PREM as our reference model (see supporting information for details). Since the two elastic moduli and density are Jeffrey's parameters [Tarantola, 2005], we parameterize the variables in common log space.

We apply perturbations in the amount of $\Delta \mathbf{m}_{p}^{j}=\log _{10}(1.01)$ to the two elastic moduli and density of the reference model independently while holding the other two parameters fixed, thus generating three new Earth models that are perturbed with respect to PREM. The perturbation of $\Delta \mathbf{m}_{p}^{j}=\log _{10}(1.01)$, 

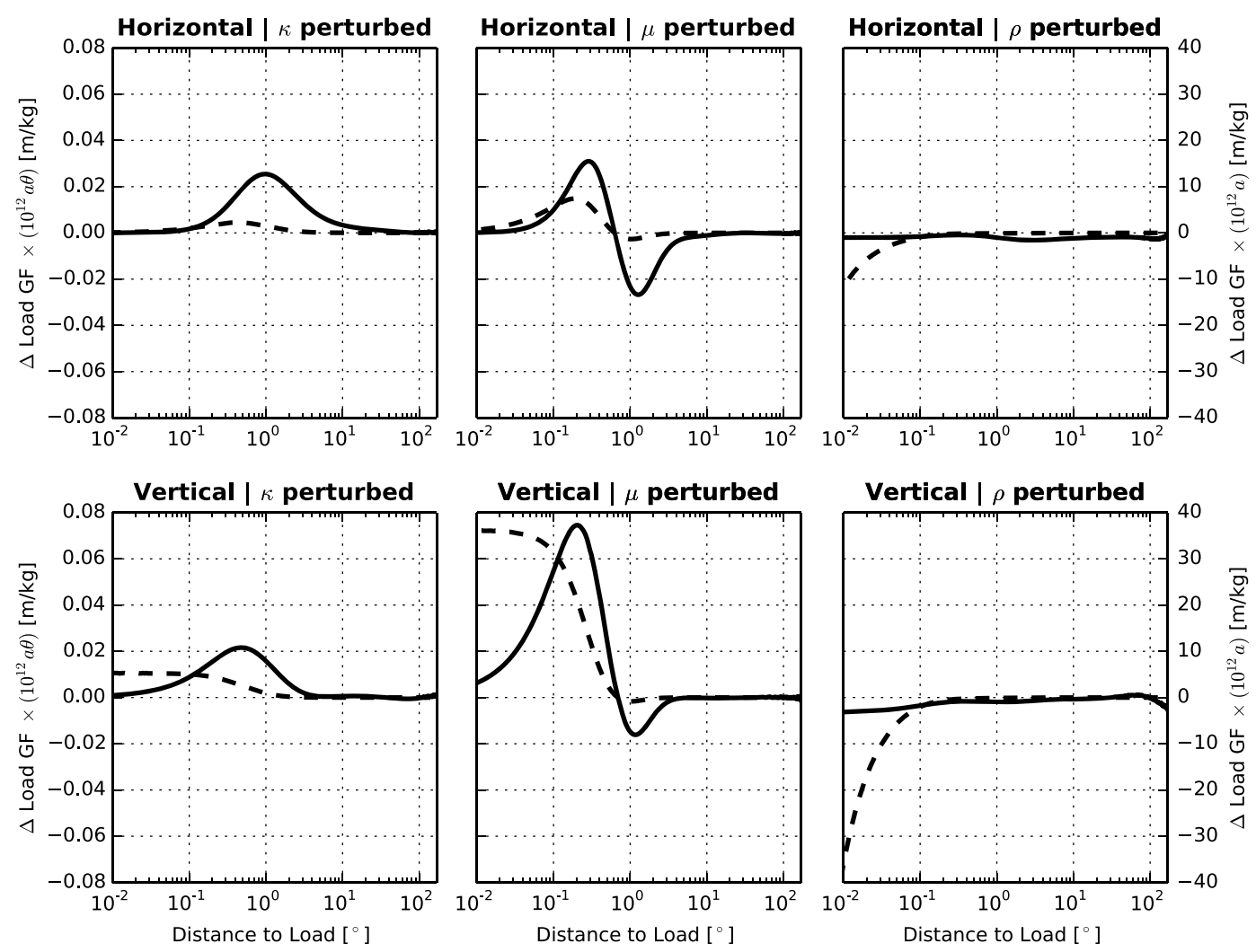

Figure 5. Direct differences between displacement load Green's functions derived from the three perturbed Earth models shown in Figure 4 and the unperturbed PREM. (top row) The horizontal component of the displacement LGF differences. (bottom row) The vertical component of the displacement LGF differences. The perturbed models were generated by augmenting the (left column) bulk modulus, (middle column) shear modulus, and (right column) density profiles by a factor of $1 \%$ in linear space between 80 and $24.4 \mathrm{~km}$ depth (upper mantle). All panels are depicted on the same scale for comparison. The solid black lines show the direct LGF differences scaled by a factor of $10^{12} a \theta$, where $a$ is Earth's radius and $\theta$ is the angular distance between the load point and the measurement site. The axes on the left pertain to the solid black lines. The dashed black lines show the direct LGF differences scaled by a factor of $10^{12} a$ (i.e., without multiplication by the angular distance, $\theta$ ). The axes on the right pertain to the dashed black lines. Note that we depict the angular distance dependence of the LGF differences on logarithmic scales.

where $j$ represents the perturbed region from 80 to $24.4 \mathrm{~km}$ depth and $p$ represents the model parameter $(\kappa, \mu$, or $\rho$ ), corresponds to $\mathrm{a}+1 \%$ linear perturbation to the original material properties. The four Earth models (original PREM and three perturbed) are shown in Figure 4.

The four Earth models may now be used to compute four sets of displacement LGFs. The direct differences between the three perturbed and the unperturbed displacement LGFs are shown in Figure 5. We scale the displacement LGFs by a conventional factor of $10^{12} a \theta$ (solid lines in Figure 5), where $a$ is Earth's radius and $\theta$ is the angular distance between the load point and the measurement site, to mitigate the singularity at the load point and to accentuate midfield and far-field features of the LGFs that are otherwise difficult to discern.

We note, however, that multiplying the LGFs by $\theta$ can also obscure LGF differences in the near field. The near-field LGFs are particularly significant for loads approximately collocated with a measurement site, such as in the cases of local hydrological or atmospheric loading. For globally distributed loads, on the other hand, the near-field loads generally account for only a small fraction of the total load. In our development of the LGF sensitivity kernels, we typically illustrate the LGF differences using the $\theta$ scaling convention for clarity, recognizing that sensitivities in the very near field can be more substantial than the results might suggest. For comparison, we show LGF differences that are both scaled (solid lines) and unscaled (dashed lines) with respect to $\theta$ in Figure 5.

In general, a perturbation of $1 \%$ to the density structure in the upper mantle yields relatively small changes in the displacement LGFs in comparison to a perturbation of $1 \%$ to either the bulk or shear modulus (Figure 5). 
Within a few kilometers of the load point, however, the sensitivity of the LGFs to perturbations in density structure increases significantly. A perturbation to the shear modulus in the upper mantle generates both positive and negative changes in the LGFs as a function of distance to the load point, with the transition between the regimes occurring at approximately the same distance to the load point as the depth of the perturbation. Moreover, positive perturbations to the bulk modulus in the upper mantle generate predominantly positive differences between the perturbed and unperturbed LGFs. For SML-induced vertical depressions (i.e., negative-valued LGFs), a positive change in the LGFs indicates less deformation or a smaller vertical displacement.

We now expand upon the direct differences between displacement LGFs to generate sensitivity kernels from finite differences. The sensitivities of displacement LGFs to small perturbations in the elasticity and density parameters are computed according to the equation

$$
K_{p, G}^{j}(\theta)=\frac{G\left(\theta, \mathbf{m}+\Delta \mathbf{m}_{p}^{j}\right)-G(\theta, \mathbf{m})}{\Delta \mathbf{m}_{p}^{j}}=\frac{\Delta G}{\Delta \mathbf{m}_{p}^{j}}
$$

where $j$ corresponds to a particular perturbed layer, $p$ corresponds to the model parameter being perturbed $(\mu, \kappa$, or $\rho)$, and $G$ represents the displacement LGF (vertical or horizontal), which depends on Earth structure, $\mathbf{m}$, and the angular distance between the load and the observer, $\theta$. The perturbation to structure, $\Delta \mathbf{m}_{p}^{j}$ involves a perturbation to the model parameter $p$ in layer $j$; all other model parameters remain unperturbed.

In comparison, Ito and Simons [2011] defined their sensitivity kernels as a percentage difference between the perturbed and unperturbed LGFs, computed in response to $1 \%$ perturbations to the elastic structure. It is also worth noting that the sensitivity kernels, $K_{p, G^{\prime}}^{j}$ depend on the thickness of the perturbed layer, $j$, and that equation (12) does not explicitly normalize by layer thickness. Thus, we document specifically the layer thicknesses used throughout our analysis. Recall also that since we have assumed a SNREI Earth structure, all perturbations are made to spherically symmetric shells.

Figure 6 shows displacement LGFs and their corresponding sensitivity kernels, scaled by $10^{12} a \theta$, for perturbations to the bulk modulus ( $c$ and d), shear modulus ( $e$ and $f$ ), and density ( $g$ and $h$ ) for each of the major PREM regions above the core. We have adopted the CM reference frame for the LGF computations in order to remain consistent with conventional GNSS analysis. We note, however, that the sensitivity kernels are reference frame independent with the exception of the density kernel, which exhibits minor sensitivity differences between reference frames due to the change in total Earth mass associated with the perturbation. Since several layers are concentrated within the top $200 \mathrm{~km}$ of the model space, we provide a zoomed-in version of Figure 6 in the supporting information.

We find that the magnitudes of the LGF sensitivities to perturbations in the elastic moduli are greatest when the perturbations are applied to near-surface structure and diminish with perturbations to deeper layers, even for perturbations to layers of far greater thickness than the shallowest layers. Perturbing a region as expansive as the central lower mantle (2741-771 km depth), for example, yields peak sensitivities approximately an order-of-magnitude smaller than the estimated peak sensitivities for perturbations to much thinner, near-surface layers.

Furthermore, the peak sensitivity to perturbations in the elastic moduli occurs farther from the load point as the perturbation depth increases. Perturbations to near-surface structure predominantly affect the LGFs in the near field, whereas perturbations to deeper structure have increasing influence over the LGFs at angular distances farther from the load point. The characteristic "moveout" of the sensitivity kernels for the bulk and shear moduli could be predicted from the Love number analysis: the high-degree LLNs, which largely define the displacement LGFs in the near field, are predominantly sensitive to near-surface structure (e.g., Figure 3). The sensitivity kernels for the bulk and shear moduli exhibit strong resemblance, except the shear modulus sensitivity transitions between positive and negative regimes, whereas the bulk modulus sensitivity remains mostly positive.

The density kernels, in contrast, exhibit peak sensitivities at short angular distances between the load point and the measurement site, regardless of perturbation depth. Moreover, perturbations to the thick central lower mantle generate the largest load-induced displacement differences. The observations are consistent with an increase to the total Earth mass that enhances the gravitational force on the applied mass load. 

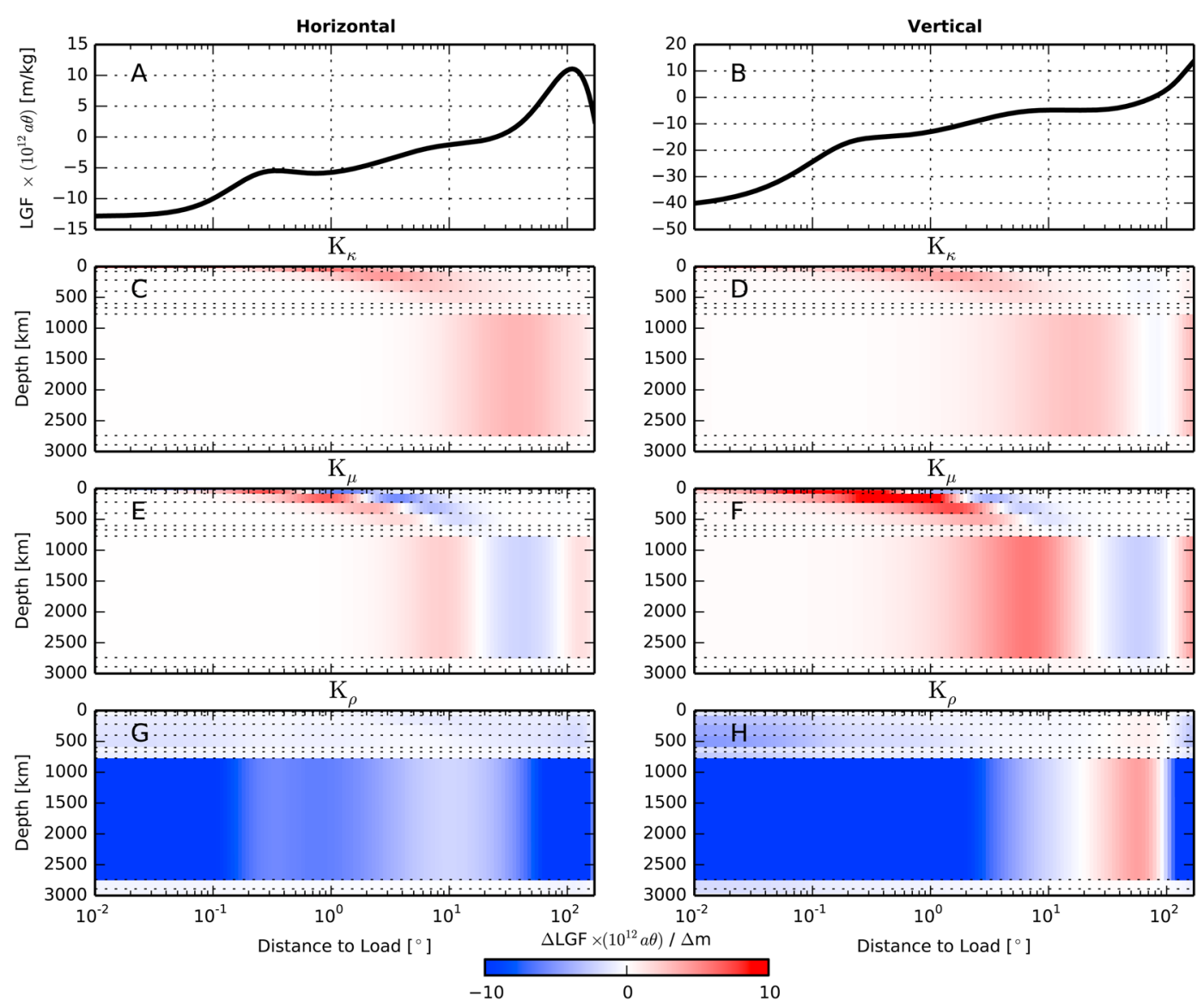

Figure 6. The sensitivity of displacement LGFs to perturbations in elastic structure for a radially heterogeneous Earth model. We adopt an isotropic and oceanless version of PREM as the reference model. ( $a$ and $b$ ) We examine the sensitivity of the displacement LGFs to linear perturbations of $1 \%$ to the ( $c$ and $d$ ) bulk modulus, (e and $f$ ) shear modulus, and ( $\mathrm{g}$ and $\mathrm{h}$ ) density as a function of depth using equation (12). We independently perturb each of the major regions of PREM beyond the core as distinct blocks, separated by dashed lines in Figures $6 c-6 \mathrm{~h}$. The regions include the lower mantle from 2891 to $2741 \mathrm{~km}$ depth, the lower mantle from 2741 to $771 \mathrm{~km}$ depth, the lower mantle from 771 to $670 \mathrm{~km}$ depth, the transition zone from 670 to $600 \mathrm{~km}$ depth, the transition zone from 600 to $400 \mathrm{~km}$ depth, the transition zone from 400 to $220 \mathrm{~km}$ depth, the low-velocity zone (LVZ) from 220 to $80 \mathrm{~km}$ depth, the region above the LVZ (LID) from 80 to $24.4 \mathrm{~km}$ depth, the lower crust from 24.4 to $15 \mathrm{~km}$ depth, the upper crust from 15 to $3 \mathrm{~km}$ depth, and the top layer from 3 to $0 \mathrm{~km}$ depth. Model parameters are defined in common log space as $\mathbf{m}_{\mu}=\log _{10} \mu$, $\mathbf{m}_{\kappa}=\log _{10} \kappa$, and $\mathbf{m}_{\rho}=\log _{10} \rho$. The model parameter perturbation is $\Delta \mathbf{m}_{p}^{j}=\log _{10}(1.01)$. The horizontal components of the displacement LGFs and sensitivity kernels are shown in Figures $6 \mathrm{a}, 6 \mathrm{c}, 6 \mathrm{e}$, and $6 \mathrm{~g}$; the vertical components are shown in Figures $6 \mathrm{~b}, 6 \mathrm{~d}, 6 \mathrm{f}$, and $6 \mathrm{~h}$. Figures $6 \mathrm{a}$ and $6 \mathrm{~b}$ depict the displacement LGFs in the CM reference frame derived from the reference model. The displacement LGFs, as well as the sensitivity kernels, were multiplied by the factor $10^{12} a \theta$ to remove the singularity at the load point and to scale the magnitude of the response, where $a$ is Earth's radius in meters and $\theta$ is the angular distance from the load point in radians. Units of the unscaled LGFs are meters per kilogram.

The magnitude of density sensitivity, $K_{\rho, G^{\prime}}^{j}$ as a function of angular distance between load and observer, $\theta$, tends to follow the pattern of the original displacement LGF. In general, the larger the magnitude of a displacement LGF at a particular angular distance, the larger the LGF sensitivity will be for a given density perturbation.

Since the layer thicknesses differ significantly among the regions of the PREM model, we also computed the LGF sensitivities for perturbations to layers of constant thicknesses. Again, using the isotropic and oceanless version of PREM as our reference model, we subdivided the crust and mantle into $20 \mathrm{~km}$ thick layers, or spherical shells, down to a depth of $800 \mathrm{~km}$. Figure 7 depicts LGF sensitivities derived from perturbations to the $20 \mathrm{~km}$ thick shells. We perturbed the model parameters by an amount of $\Delta \mathbf{m}_{p}^{j}=\log _{10}(1.01)$. With the thickness of the layers held fixed, we find that the magnitude of peak sensitivity decreases with perturbation depth for the two elastic moduli and that most of the sensitivity stems from perturbations applied to the Earth's 

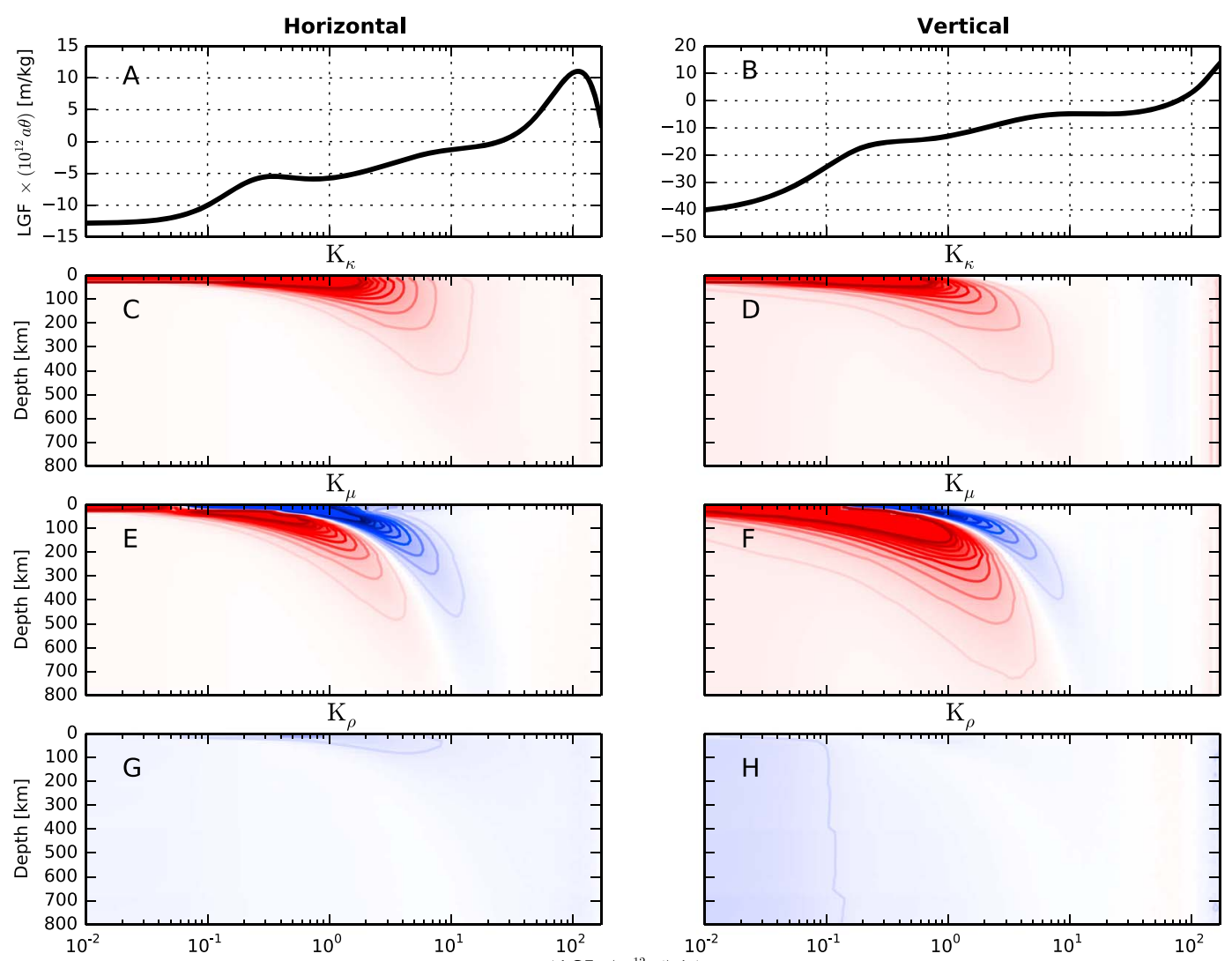

Distance to Load $\left[{ }^{\circ}\right]$
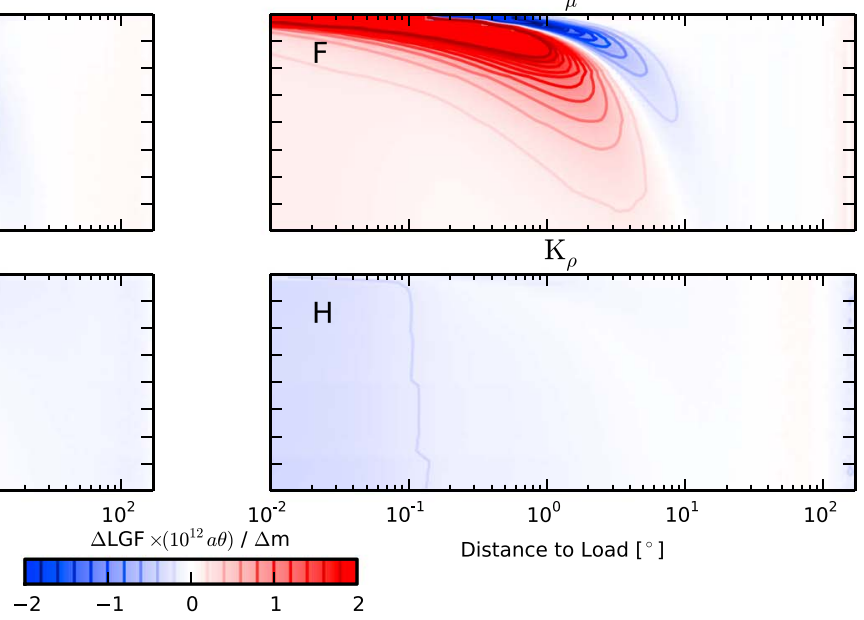

$\mathrm{H}$

Figure 7. Same as Figure 6 except that we have perturbed layers of constant thickness instead of the major regions of PREM. Specifically, we have partitioned the crust and mantle into a set of $20 \mathrm{~km}$ thick spherical shells, which we perturb systematically down to $800 \mathrm{~km}$ depth. Again, we adopted a model parameter perturbation of $\Delta \mathbf{m}_{p}^{j}=\log _{10}(1.01)$. Contour lines are included for clarity, with specific values denoted in the color bar.

outermost $500 \mathrm{~km}$. The density sensitivity, on the other hand, remains relatively constant as a function of perturbation depth. Furthermore, the sensitivity to $1 \%$ perturbations in density structure is significantly lower than the sensitivity to $1 \%$ perturbations in elastic structure. The sensitivity kernels for perturbations to $50 \mathrm{~km}$ thick spherical shells are provided in the supporting information.

To examine variations in the LGF sensitivities as a function of perturbation depth, Figure 8 depicts slices through the sensitivity diagrams from Figure 7 at an angular distance of $2.5^{\circ}$ from the applied load. The range of the density sensitivity is far smaller than the sensitivity ranges for the two elastic moduli and, again, illustrates that the kernel remains approximately constant in magnitude regardless of the depth at which density is perturbed. The profiles also show that the density kernel remains mostly negative, the bulk modulus kernel remains mostly positive, and the shear modulus kernel exhibits both positive and negative sensitivity, depending on the perturbation depth. Note as well that the sensitivities are strongest within about $250 \mathrm{~km}$ depth from the surface, which is approximately equivalent to the distance of $2.5^{\circ}$ between the profile line and the load point [cf. Okubo, 1988b].

For completeness, we acknowledge that alternative approaches exist for defining the model parameters. For example, rather than defining the model parameters in terms of common logarithms of the elastic moduli and density directly, one could instead use ratios of the elastic properties: $\mathbf{m}_{\mu}^{j}=\log _{10} \frac{\mu_{j+1}}{\mu_{j}}, \mathbf{m}_{\kappa}^{j}=\log _{10} \frac{\kappa_{j+1}}{\kappa_{j}}$, and $\mathbf{m}_{\rho}^{j}=\log _{10} \frac{\rho_{j+1}}{\rho_{j}}$. Reference values $\left(\mu_{0}, \kappa_{0}\right.$, and $\left.\rho_{0}\right)$ must necessarily be defined for the elastic properties of the starting layer, which may be either at the top or at the base of the model space in depth. According to this definition, LGF sensitivities would be computed based on perturbations to interface contrasts, rather than based on perturbations to distinct layers. 

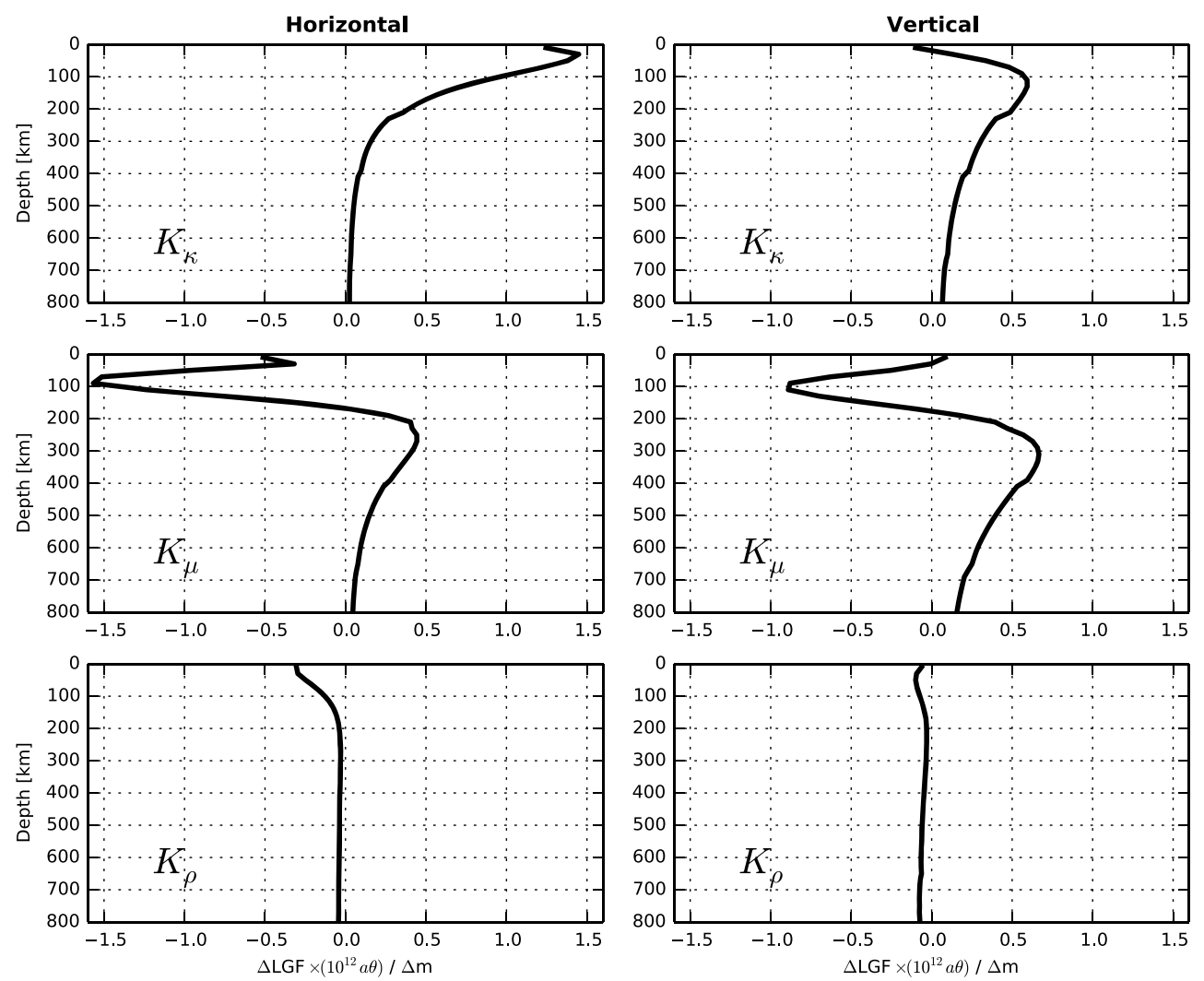

Figure 8. Sensitivity kernels for the displacement LGFs at an angular distance of $2.5^{\circ}$ from the load point. The kernels depict slices through the sensitivity diagrams in Figure 7 at $\theta=2.5^{\circ}$.

Furthermore, building upon the Love number partial derivatives, LGF sensitivities may also be computed quasi-analytically [Okubo, 1988a, 1988b]. The methodology combines the Love number partial derivatives already developed with Legendre polynomials in large algebraic expansions analogous to equations (5) and (6). We defer a more complete analysis and description of the quasi-analytical LGF sensitivities to the future.

Finally, we consider the sensitivity of displacement LGFs to a variety of standard Earth models in order to obtain a general sense for the range of acceptable structural perturbations and LGF differences expected for a SNREI Earth. The models that we consider here include the following: PREM, STW105 [Kustowski et al., 2008], AK135f [Kennett et al., 1995], SNA [Grand and Helmberger, 1984], CR [Chu et al., 2012], and 1066A. AK135f represents the radially symmetric AK135 seismic velocity model of Kennett et al. [1995], supplemented by the density model of Montagner and Kennett [1996]. PREM, STW105, AK135f, and 1066A represent globally averaged structure, whereas CR and SNA represent regional cratonic and stable North American structures, respectively. Below approximately $1000 \mathrm{~km}$ depth, both CR and SNA assume the structural properties of AK135f. For PREM, STW105, and AK135f, we replaced the water layer at the surface with typical values for the upper crust: $V_{P}=5800 \mathrm{~m} \mathrm{~s}^{-1}, V_{S}=3200 \mathrm{~m} \mathrm{~s}^{-1}$, and $\rho=2600 \mathrm{~kg} \mathrm{~m}^{-3}$. Tables of the reference Earth models used in this study are provided in the supporting information (Data Sets S1-S6).

Figure 9 shows profiles of the elastic moduli and density for the six reference Earth models as well as the deviation of each model from PREM. Discrepancies between the SNREI models are largest in the crust and upper mantle, primarily due to variations in crustal properties and different definitions of the Moho depth. In general, however, the differences are less than 0.05 , or $\approx \log _{10}(1.1)$, which corresponds to variations in $\kappa, \mu$, and $\rho$ (in linear space) of about $10 \%$ or less at a given depth.

Figure 10 shows displacement LGFs derived from each of the SNREI Earth models depicted in Figure 9. The LGFs in Figures $10 \mathrm{c}-10 \mathrm{~h}$ were multiplied by the factor $10^{12} a \theta$, whereas the LGFs in Figures 10a and $10 \mathrm{~b}$ remain unscaled with respect to $\theta$. When the LGFs remain unscaled with respect to $\theta$, the diminishing 

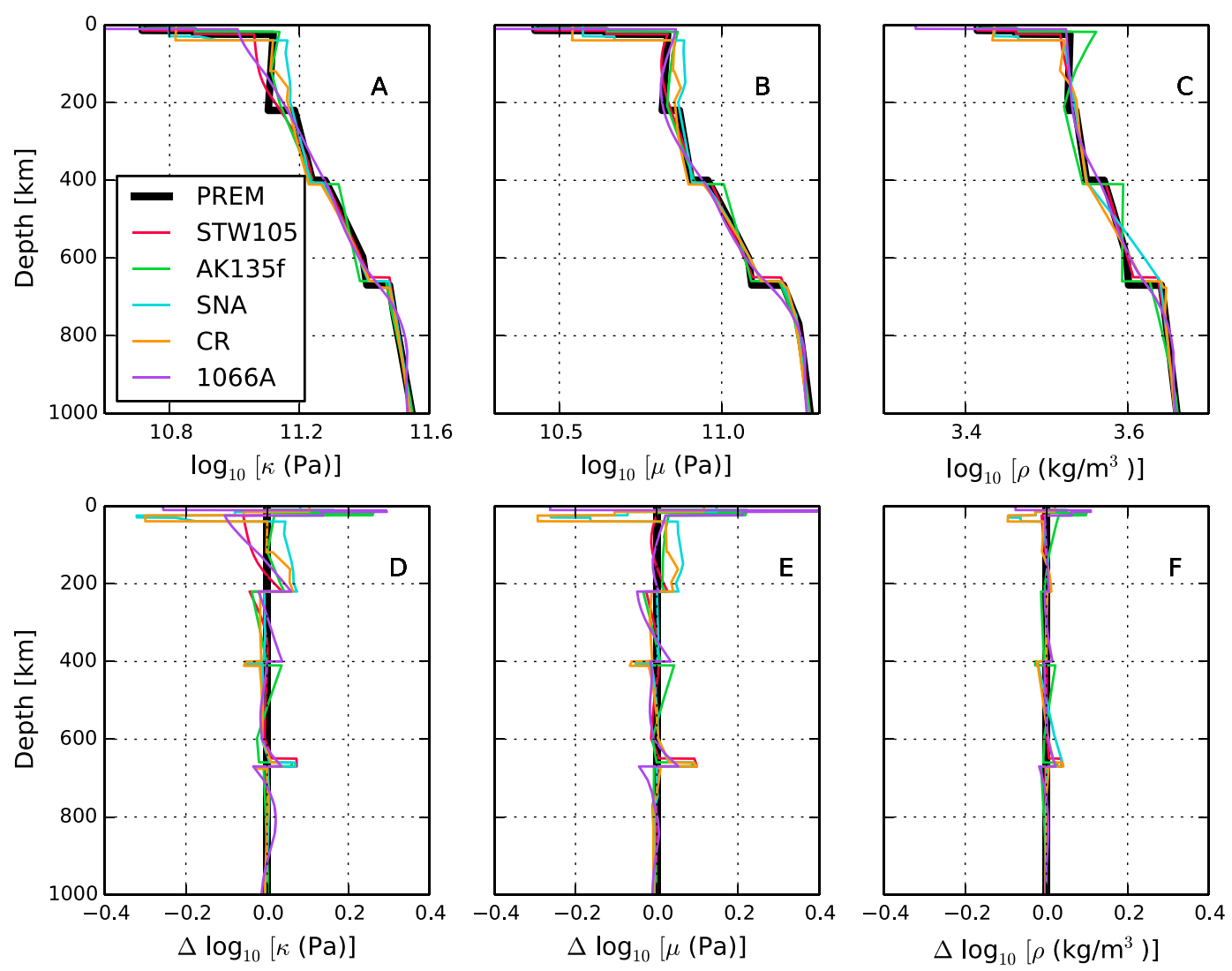

Figure 9. A comparison between six seismologically derived Earth models: PREM (black), STW105 (red), AK135f (green), SNA (blue), CR (orange), and 1066A (purple). All models are assumed spherically symmetric, nonrotating, elastic, and isotropic (SNREI). The SNA and CR models, derived for stable North America and cratonic structures, respectively, assume a structure equivalent to AK135f below $\sim 1000 \mathrm{~km}$ depth. For more complete descriptions of the models, see the supporting information. (a-c) Profiles of the bulk modulus $(\kappa)$, shear modulus $(\mu)$, and density $(\rho)$ in log space. $(d-f)$ The deviation of each model from PREM (in log space) as a function of depth.

amplitudes of the load-induced displacements as a function of angular distance away from the load point are more apparent. Tables of the displacement LGFs and LLNs derived from the six reference Earth models are provided in the supporting information (Data Sets S7-S24).

Based on the direct differences between the displacement LGFs shown in Figures $10 \mathrm{~g}$ and $10 \mathrm{~h}$, we infer that the vertical displacement sensitivities are generally larger in magnitude than the horizontal displacement sensitivities. Sensitivities computed as a percentage difference of the reference LGF rather than a direct difference, however, are generally larger for the horizontal displacement component [lto and Simons, 2011]. The LGFs associated with Earth model 1066A exhibit the largest differences with respect to PREM, particularly in the near field (within $0.1^{\circ}$ of the load point), mostly due to significant differences in the material properties of the upper crust.

In general, the LGF sensitivities for the reference Earth models tend to be largest at measurement sites within $\sim 1^{\circ}$ of the load, and taper off substantially beyond $10^{\circ}$, even with perturbations to deep structure. Since we defined the globally averaged models PREM, STW105, and AK135f to have the same upper crustal structure, the LGF differences between the models are very small in the near field and decrease toward zero as the angular distance between load and observer, $\theta$, approaches zero. Even small perturbations to upper crustal layers, however, can yield large variations in the LGFs at short observer-to-load angular distances [cf. Wang et al., 2012; Dill et al., 2015; Bos et al., 2015], as exemplified by the high-degree LLNs shown in Figure 3, the LGF differences between 1066A and PREM, and the LGF differences between the region-specific (CR and SNA) and globally averaged Earth models (PREM, STW105, and AK135f). 

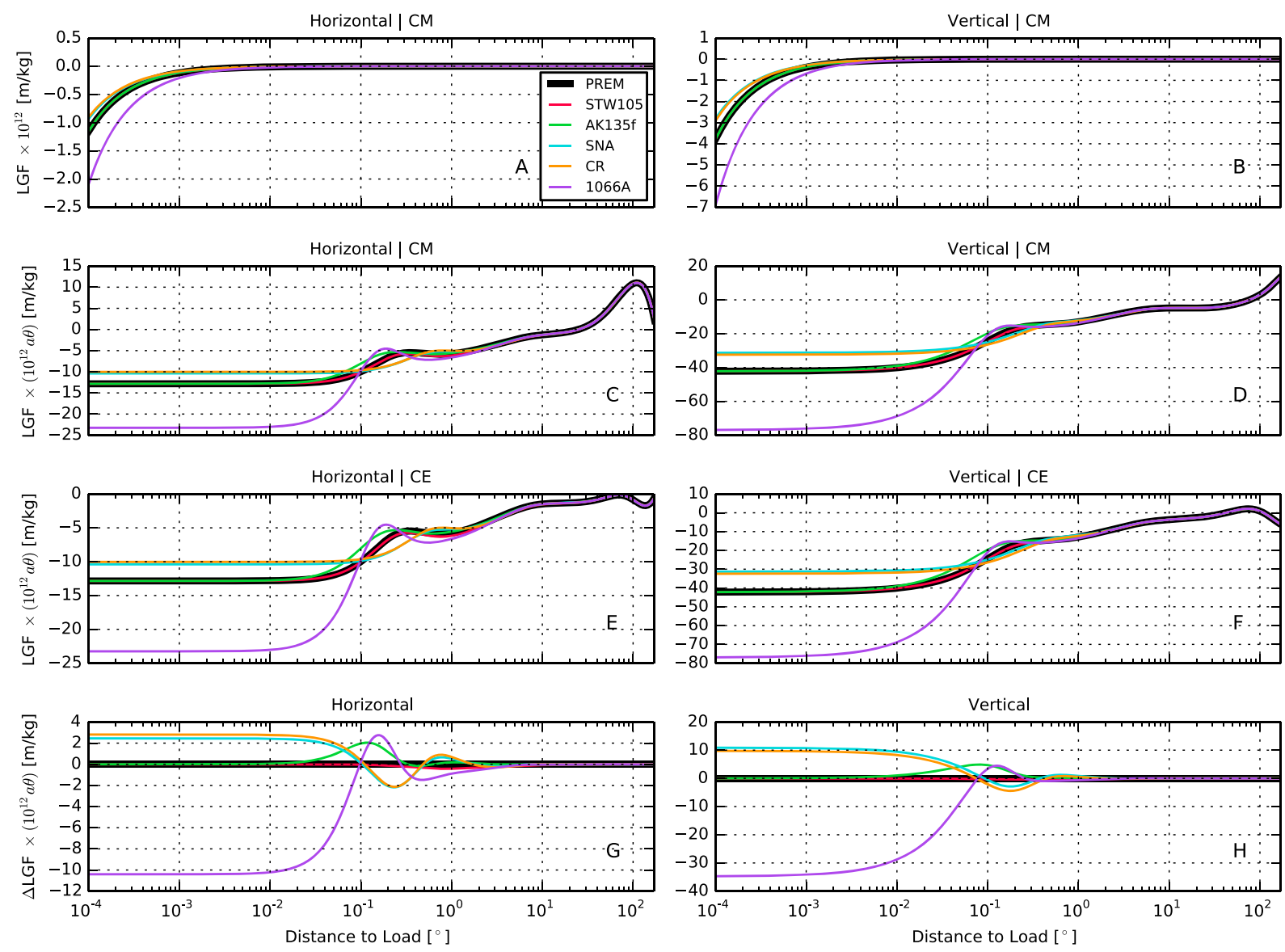

Figure 10. Displacement LGFs for various seismologically derived Earth models (Figure 9). The (a) horizontal and (b) vertical components of the displacement LGFs, respectively, in the CM reference frame. (c and d) Same as Figures 10a and 10b, respectively, but multiplied by an additional factor of $a \theta$, where $a$ is Earth's radius and $\theta$ is the angular separation between the load point and the observer. The (e) horizontal and (f) vertical components of the displacement LGFs, respectively, in the CE reference frame. ( $g$ and $h$ ) The differences between the horizontal and vertical displacement LGFs, respectively, relative to the displacement LGFs derived from PREM. Since the total Earth mass varies only slightly between the different Earth models, the dependence of the LGF differences on reference frame is negligible.

\subsection{Predicted OTL-Induced Surface Displacements}

So far, we have explored the patterns of load-induced surface displacements caused by point loads of unit mass. In reality, surface mass loads are not point sources but rather distributed across regional and global scales. Furthermore, the mass density of a load can be highly spatially variable. Whereas the sensitivity kernels for the displacement LGFs are characterized in terms of perturbation depth and the angular distance between the point load and the measurement site, sensitivity kernels for the surface displacements induced by a load of finite size are specific to the location of a measurement site in relation to the entire, distributed load.

Predicting the surface displacements generated by a spatially variable mass load of finite size requires a convolution of displacement LGFs with the load model (equations (8) and (9)). The predicted displacements depend on the spatial and temporal characteristics of the load, the LGFs derived for a particular Earth structure, and the location of the measurement site relative to the load. For the special case of OTL, measurement sites located directly adjacent to large-amplitude tides offshore tend to exhibit relatively large OTL-induced surface displacements (Figure 10 and equation (8)).

As an example, we consider OTL-induced surface displacements generated by the principal lunar semidiurnal $\left(M_{2}\right)$ tidal harmonic. Figure 11 shows a map of Iceland surrounded by the $M_{2}$ ocean tide from the TPXO8-Atlas model, which was constrained in part by empirical data from multiple satellite altimetry missions and validated against local tide gauge measurements [Egbert and Erofeeva, 2002; Egbert et al., 2010]. Predicted surface displacements, produced by a convolution of the TPXO8-Atlas tide model with displacement LGFs based on PREM, are overlain on the map and depicted as particle motion ellipses (PMEs). Each of the PMEs, which are 


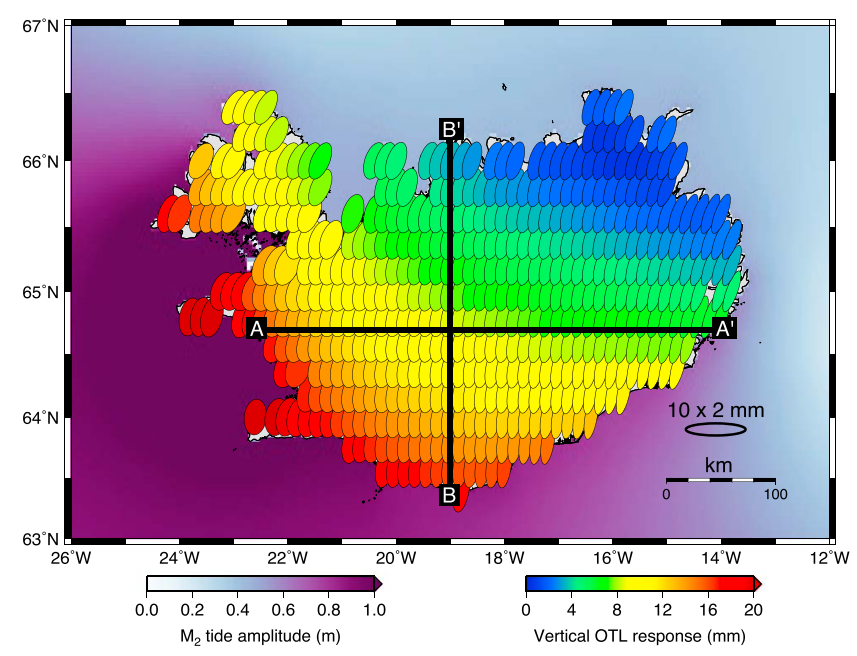

Figure 11. Predicted OTL-induced surface displacements for the $M_{2}$ tidal harmonic, derived from the TPXO8-Atlas ocean tide model [Egbert and Erofeeva, 2002; Egbert et al., 2010] and PREM, shown as particle motion ellipses (PMEs) on a $0.2^{\circ} \times 0.2^{\circ}$ grid across Iceland. The size and orientation of each ellipse represent the horizontal displacement response; the color of each ellipse represents the vertical displacement response (right color bar). A reference ellipse for the horizontal motion is provided in the lower right corner of the figure. The left color bar depicts the $M_{2}$ tide amplitude in the oceans. Two profile lines $\left(A-A^{\prime}\right.$ and $\left.B-B^{\prime}\right)$ are superimposed. In the subsequent figures, we explore changes in predicted OTL-induced surface displacements due to small perturbations in the elastic structure of the crust and mantle at high spatial resolution along the profile lines (Figures 15, 16, S21, and S22).

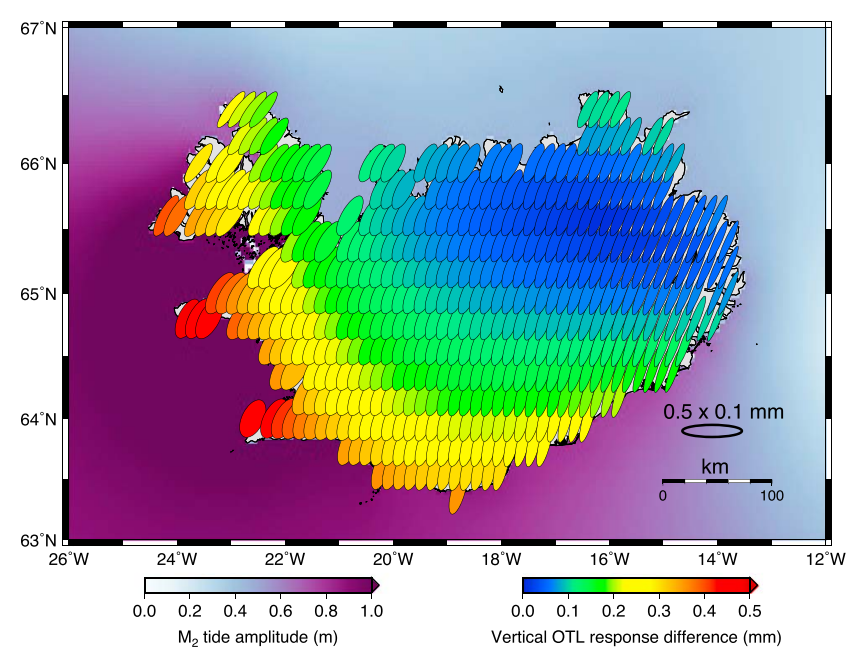

Figure 12. Vector differences between pairs of predicted $M_{2}$ OTL-induced surface displacements derived from PREM and STW105, shown as PMEs on a $0.2^{\circ} \times 0.2^{\circ}$ grid across Iceland. The size and orientation of each ellipse represent the differential horizontal displacement response; the color of each ellipse represents the differential vertical displacement response (right color bar). A reference PME for the horizontal motion is provided in the lower right corner of the figure. Note the change in scale of the PMEs relative to Figure 11. The left color bar depicts the $M_{2}$ tide amplitude in the oceans. centered on the geographic locations of the prediction sites $\left(0.2^{\circ} \times 0.2^{\circ}\right.$ resolution), depicts the displacement of Earth's surface due to the $\mathrm{M}_{2}$ ocean tide. Each ellipse is traced out completely during a single tidal period of $12.42 \mathrm{~h}$.

We selected Iceland as a case study for several reasons: (1) Iceland is sufficiently small to facilitate the computation of many sets of predicted OTL-induced surface displacements along very high resolution profiles that span from coast to coast; (2) the island is sufficiently large to examine differences in the predicted surface displacements even a couple hundred kilometers inland of the coast; (3) the amplitude of the $M_{2}$ tide is relatively large off the southwest shore of the island; (4) the amplitude of the $\mathrm{M}_{2}$ tide is asymmetrical about the island, which allows for an examination of the effect of tide amplitude on response sensitivity, notwithstanding distance to the coast; and (5) Iceland is a place of great geophysical interest that could benefit from future tomographic inversions of observed deformation from OTL.

Figure 12 shows the vector differences between pairs of $M_{2}$ OTL-induced surface displacements across Iceland derived from PREM and STW105. On the western coast, where the tide heights are largest, the vector differences between predicted displacements reach about $0.5 \mathrm{~mm}$. Note that since we use the same ocean tide model and convolution procedure to generate each set of predictions, errors in the ocean tide model and convolution scheme effectively cancel out when the predicted displacements are differenced. As mentioned previously, we estimate that the maximum quadrature error associated with computing the vector differences between pairs of predicted surface displacements, $\left|U_{1}-U_{2}\right|$, is on the order of a fraction of a micron.

For a $2^{\circ} \times 2^{\circ}$ global grid of land-based locations (coarsened relative to the Iceland grid for reasons of computational 

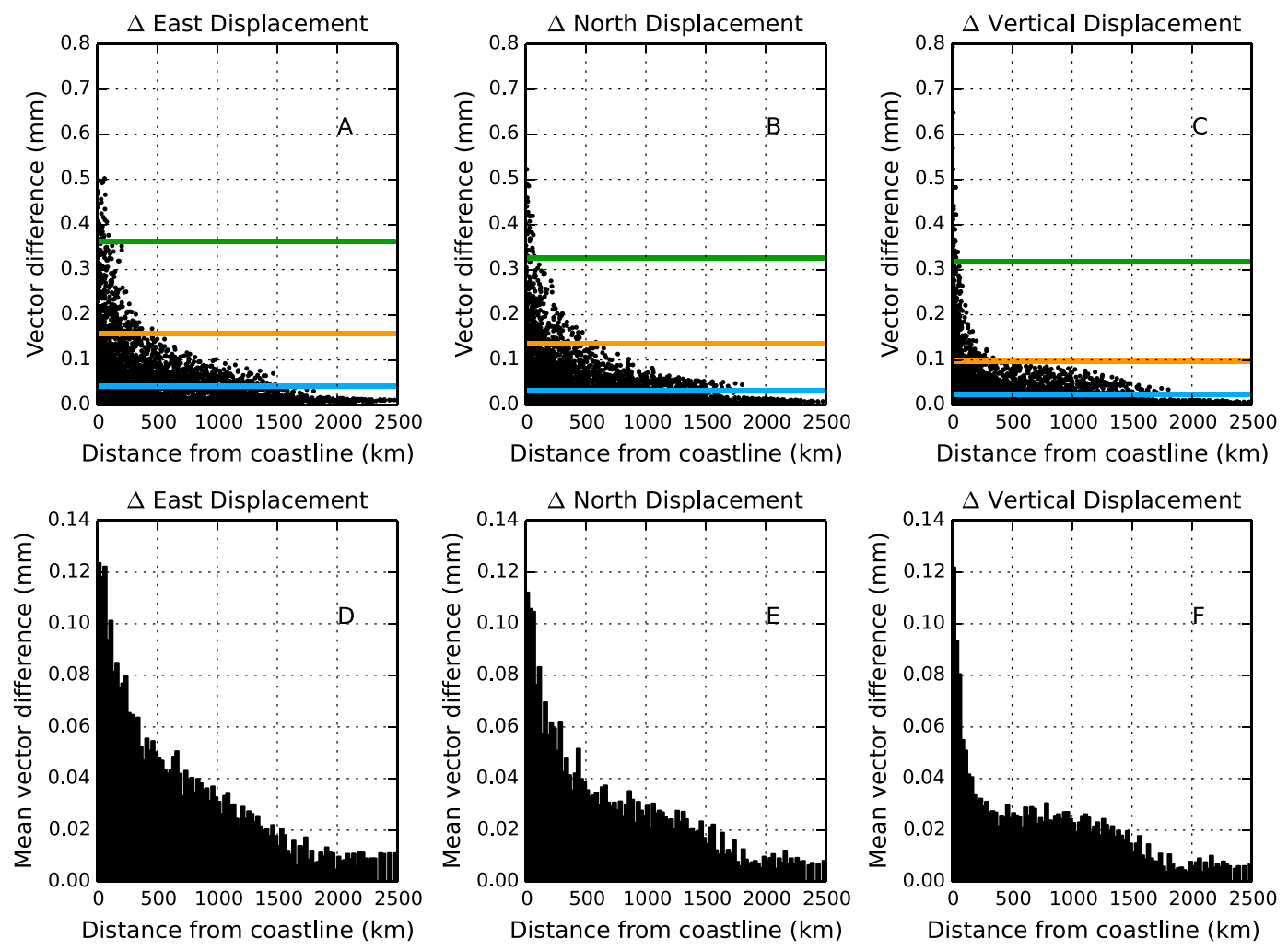

Figure 13. Vector differences between pairs of predicted $M_{2}$ OTL-induced surface displacements for PREM and STW105 as a function of distance to the nearest coastline. The two sets of predicted OTL-induced surface displacements (one set for PREM and one set for STW105) were computed on a $2^{\circ} \times 2^{\circ}$ global grid of land-based locations. Only the SNREI Earth model changes between the forward model computations; all other parameters, including the ocean tide model and convolution procedure, remain the same. The (a) east, (b) north, and (c) vertical components, respectively, of the vector differences between the pairs of predictions. The black dots indicate the vector differences at individual grid nodes. The horizontal lines in each panel represent the 50th (blue), 90th (orange), and 99th (green) percentiles of the vector differences. $(d-f)$ The mean vector differences as a function of distance to the coastline, computed in $25 \mathrm{~km}$ bins.

efficiency), $90 \%$ of the predicted $\mathrm{M}_{2}$ OTL-induced surface displacements fall below about 3.1, 3.5, and $10.9 \mathrm{~mm}$ in the east, north, and vertical components, respectively. Figure 13 shows the vector differences between pairs of predicted $\mathrm{M}_{2}$ OTL-induced surface displacements derived from PREM and STW105 across the global grid as a function of distance to the nearest coastline. Histograms showing the magnitudes of the vector differences for additional SNREI-model pairs (Figure 9) are provided in the supporting information. Even for prediction sites located within $25 \mathrm{~km}$ of a coastline, the mean vector difference between the PREM predictions and the STW105 predictions is only about $0.1 \mathrm{~mm}$. For prediction sites located very near to the coastline and in close proximity to large-amplitude tides, however, vector differences between the OTL-induced surface displacements can reach several tenths of a millimeter or more (Figure 12). Global maps of the vector differences between pairs of predicted OTL-induced surface displacements derived from PREM and STW105 are provided in the supporting information.

Since the reference Earth models (Figure 9) vary irregularly as a function of depth and model parameter, we also explore changes in OTL-induced surface displacements generated by systematic perturbations to elastic and density structure. Specifically, we investigate the effects of independent perturbations to the two elastic moduli and density on $\mathrm{M}_{2}$ OTL-induced surface displacements along two high-resolution $\left(0.01^{\circ}\right.$ or $\left.\approx 1 \mathrm{~km}\right)$ profiles through Iceland (Figure 11). One of the profiles spans from west to east across the island along $64.7^{\circ} \mathrm{N}$ latitude $\left(A-A^{\prime}\right)$; the second profile spans from south to north across the island along $341^{\circ} E$ longitude $\left(B-B^{\prime}\right)$.

As with the displacement LGFs, we first consider the direct differences between OTL-induced surface displacements derived from the three perturbed models in Figure 4 and unperturbed PREM. We define our model parameters in common log space: $\mathbf{m}_{\mu}=\log _{10} \mu, \mathbf{m}_{\kappa}=\log _{10} \kappa$, and $\mathbf{m}_{\rho}=\log _{10} \rho$. Furthermore, we compute 

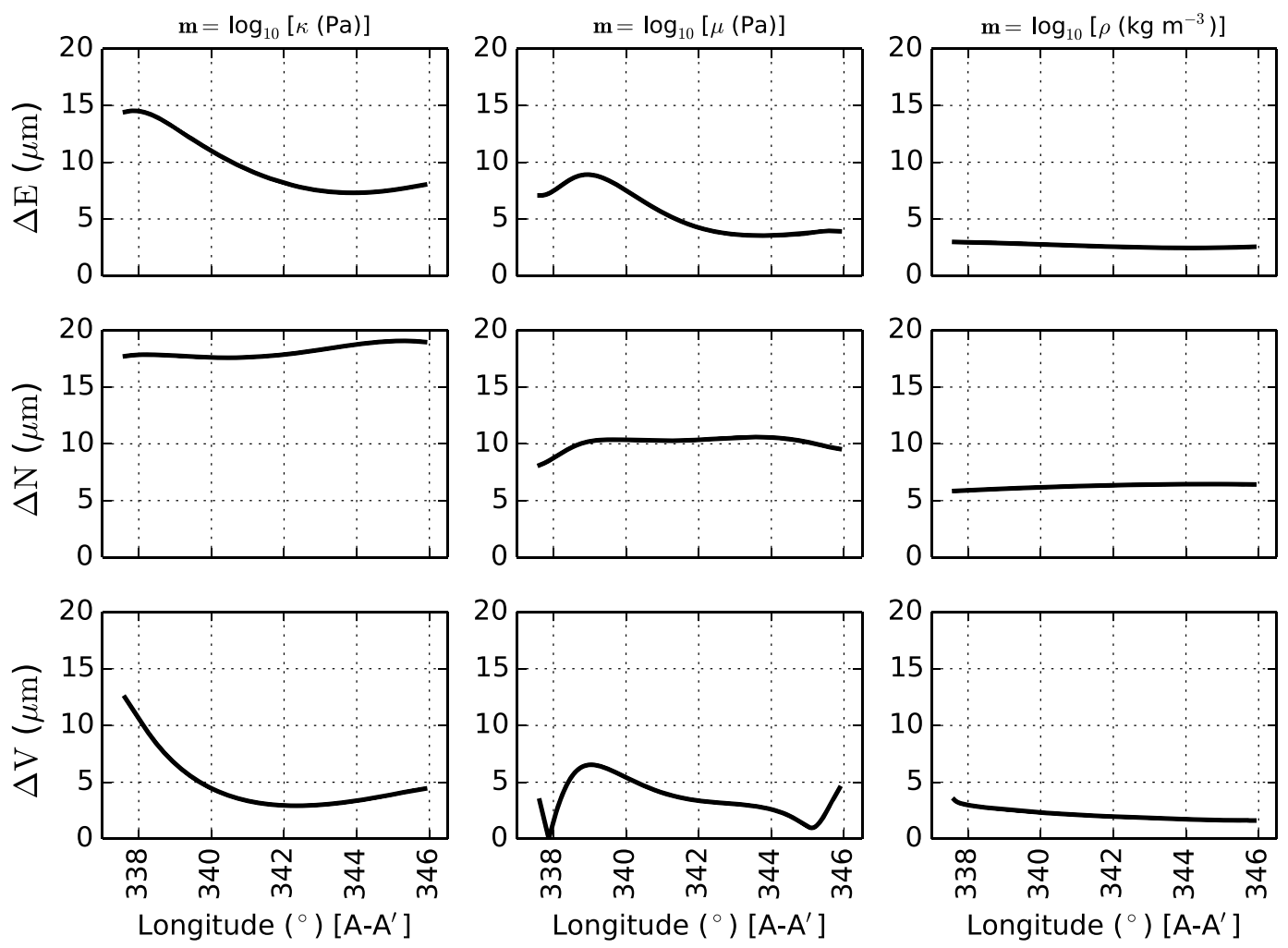

Figure 14. Magnitudes of the vector differences between $M_{2}$ OTL-induced surface displacements derived from the three perturbed Earth models shown in Figure 4 and the unperturbed PREM along the profile A- $\mathrm{A}^{\prime}$ in Figure 11 at $0.01^{\circ}$ $(\approx 1 \mathrm{~km})$ resolution. (left column) The displacement differences between the $\kappa$-perturbed model and the unperturbed PREM. (middle column) The displacement differences between the $\mu$-perturbed model and the unperturbed PREM. (right column) The displacement differences between the $\rho$-perturbed model and the unperturbed PREM. (top row) East, (middle row) north, and (bottom row) vertical components of the differential displacements. The perturbed models were generated by augmenting the bulk modulus (left), shear modulus (middle), and density (right) by a factor of $1 \%$ in linear space between 80 and $24.4 \mathrm{~km}$ depth (upper mantle). All panels are depicted on the same scale for comparison.

the response differences based on perturbations to the model parameters of $\Delta \mathbf{m}_{p}^{j}=\log _{10}(1.01)$, which correspond to $+1 \%$ linear perturbations to the original elastic parameters.

For each prediction site along the high-resolution profile $A-A^{\prime}$ from Figure 11, the magnitudes of the vector differences between the predicted OTL-induced surface displacements derived from the perturbed Earth models and the unperturbed PREM are shown in Figure 14. The procedure is analogous to the methods used to develop Figure 5 , although we now consider the finite-sized $\mathrm{M}_{2}$ OTL rather than a point source load of unit mass. For perturbations of $1 \%$ to the elastic moduli and density, the magnitudes of the vector differences are less than $0.02 \mathrm{~mm}$. For the vertical displacement component, a perturbation to the bulk modulus generates a larger change in the induced surface displacements on the western coast than on the eastern coast presumably due to the larger tidal amplitudes along the western coast. Consistent with the displacement LGFs, the $1 \%$ perturbation to density structure yields the smallest changes to the load-induced surface displacements overall. Specific details of the displacement differences along the profile, however, can be difficult to interpret, since the displacements depend on many factors, including the spatial distribution of the load relative to the prediction site and the characteristics of the LGFs as a function of distance to each load point.

We now extend our analysis of the direct vector differences between OTL-induced surface displacements to explicit finite differences. The sensitivity kernels are given by

$$
K_{p, U}^{j}\left(r, \rho_{z}, Z\right)=\frac{U\left(r, \rho_{z}, Z, \mathbf{m}+\Delta \mathbf{m}_{p}^{j}\right)-U\left(r, \rho_{z}, Z, \mathbf{m}\right)}{\Delta \mathbf{m}_{p}^{j}}=\frac{\Delta U}{\Delta \mathbf{m}_{p}^{j}}
$$



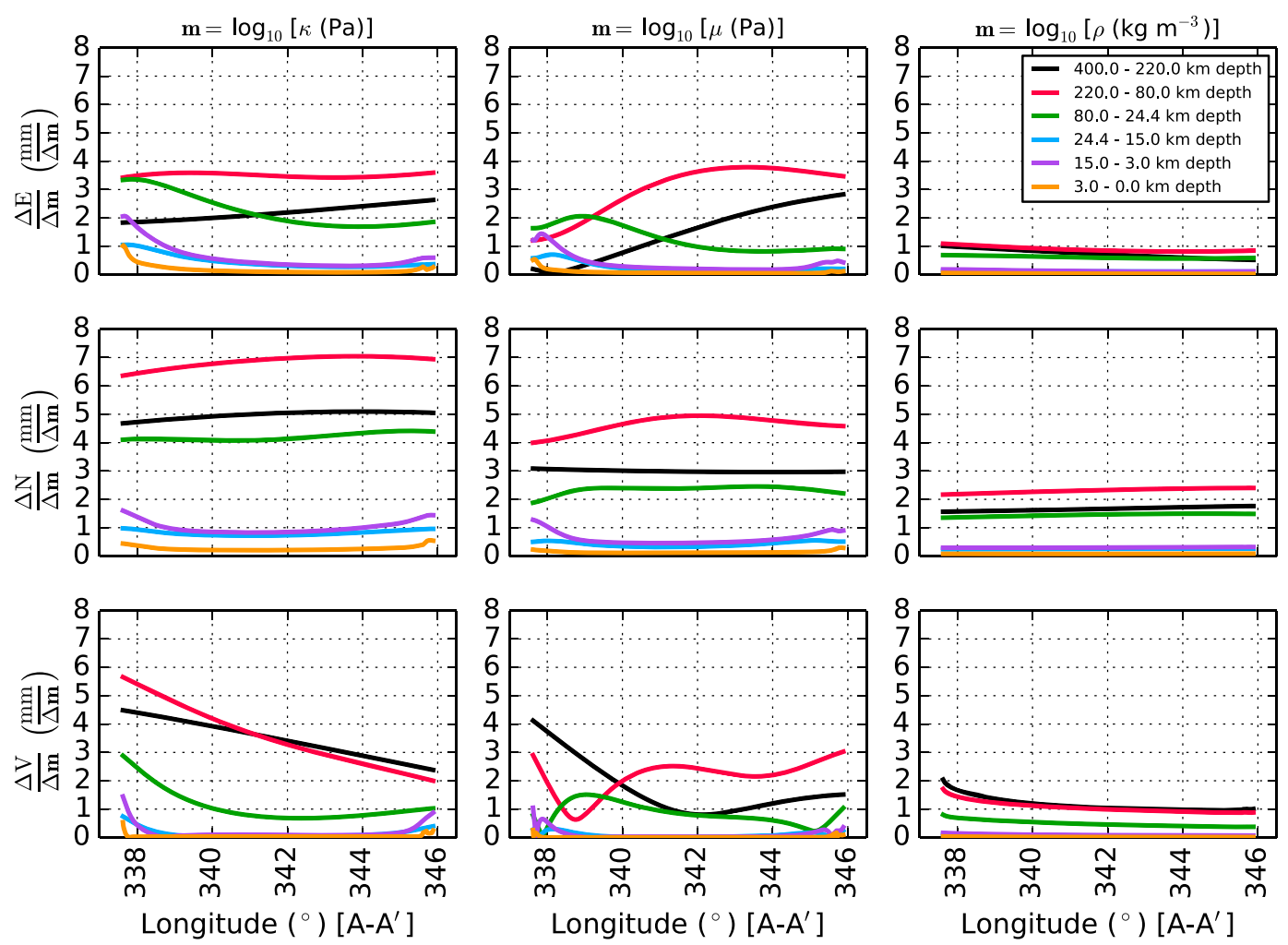

Figure 15. The sensitivity of predicted OTL-induced surface displacements to perturbations in elastic and density structure, computed along the profile $\mathrm{A}-\mathrm{A}^{\prime}$ in Figure 11. The profile maintains constant latitude at $64.7^{\circ} \mathrm{N}$ and a node spacing of $0.01^{\circ}(\approx 1 \mathrm{~km})$. (left column) The sensitivity of predicted surface displacements to perturbations in the bulk modulus model parameter, $\Delta \log _{10} \kappa$. (middle column) The sensitivity to perturbations in the shear modulus model parameter, $\Delta \log _{10} \mu$. (right column) The sensitivity to perturbations in the density model parameter, $\Delta \log _{10} \rho$. In each case, we perturb the parameters by $1 \%$ in linear space or by $\Delta \mathbf{m}=\log _{10}(1.01)$ in log space, where $\mathbf{m}=\log _{10} \kappa, \log _{10} \mu$, or $\log _{10} \rho$. Sensitivity kernels for $\mathrm{M}_{2}$ OTL-induced surface displacements in the (top row) east, (middle row) north, and (bottom row) vertical components, respectively. The colored lines denote perturbations to distinct layers of PREM down to a depth of $400 \mathrm{~km}$ and correspond to the same layer in every panel (see legend). The sensitivity kernels are computed, separately for each layer, as the magnitudes of the vector differences between the predicted OTL-induced surface displacements (in millimeters) for the perturbed and reference (unperturbed PREM) models divided by the model parameter perturbation, $\log _{10}(1.01)$.

where $j$ corresponds to a particular perturbed layer, $p$ corresponds to the model parameter being perturbed $(\mu, \kappa$, or $\rho)$, and $U$ represents a predicted OTL-induced surface displacement (equation (8)), which depends on Earth structure, $\mathbf{m}$, the load model, $Z \rho_{z^{\prime}}$ and the location of the prediction site, $r$, relative to the load. The perturbation to structure, $\Delta \mathbf{m}_{p}^{j}$, involves a perturbation to the model parameter $p$ in layer $j$; all other model parameters remain unperturbed.

Figure 15 shows the sensitivity kernels for the west-to-east profile $\left(A-A^{\prime}\right)$. The results for the south-to-north profile $\left(B-B^{\prime}\right)$ are included in the supporting information. All of the sensitivity kernels, derived from equation (13), are depicted at full resolution (i.e., $0.01^{\circ}$ spacing between prediction sites). Here we have perturbed the major regions of PREM down to $400 \mathrm{~km}$ depth. Without accounting for the thickness of each perturbed layer, we find that perturbations to relatively thick layers in the upper mantle can influence the OTL-induced surface displacements more than perturbations to relatively thin layers in the crust. In particular, the sensitivity of the surface displacements to perturbations in elastic and density structure in the upper $3 \mathrm{~km}$ of the crust is weaker overall than the sensitivity to perturbations in any other layer.

In Figure 16, we show the same sensitivity kernels depicted in Figure 15 but normalized by the thicknesses of the perturbed layers. Normalization by layer thickness allows us to better explore the effects of perturbation depth on the OTL-induced surface displacements. Particularly for the sensitivity of the displacements to perturbations in the shear modulus, it is apparent from Figure 16 that the location of the peak sensitivity as 

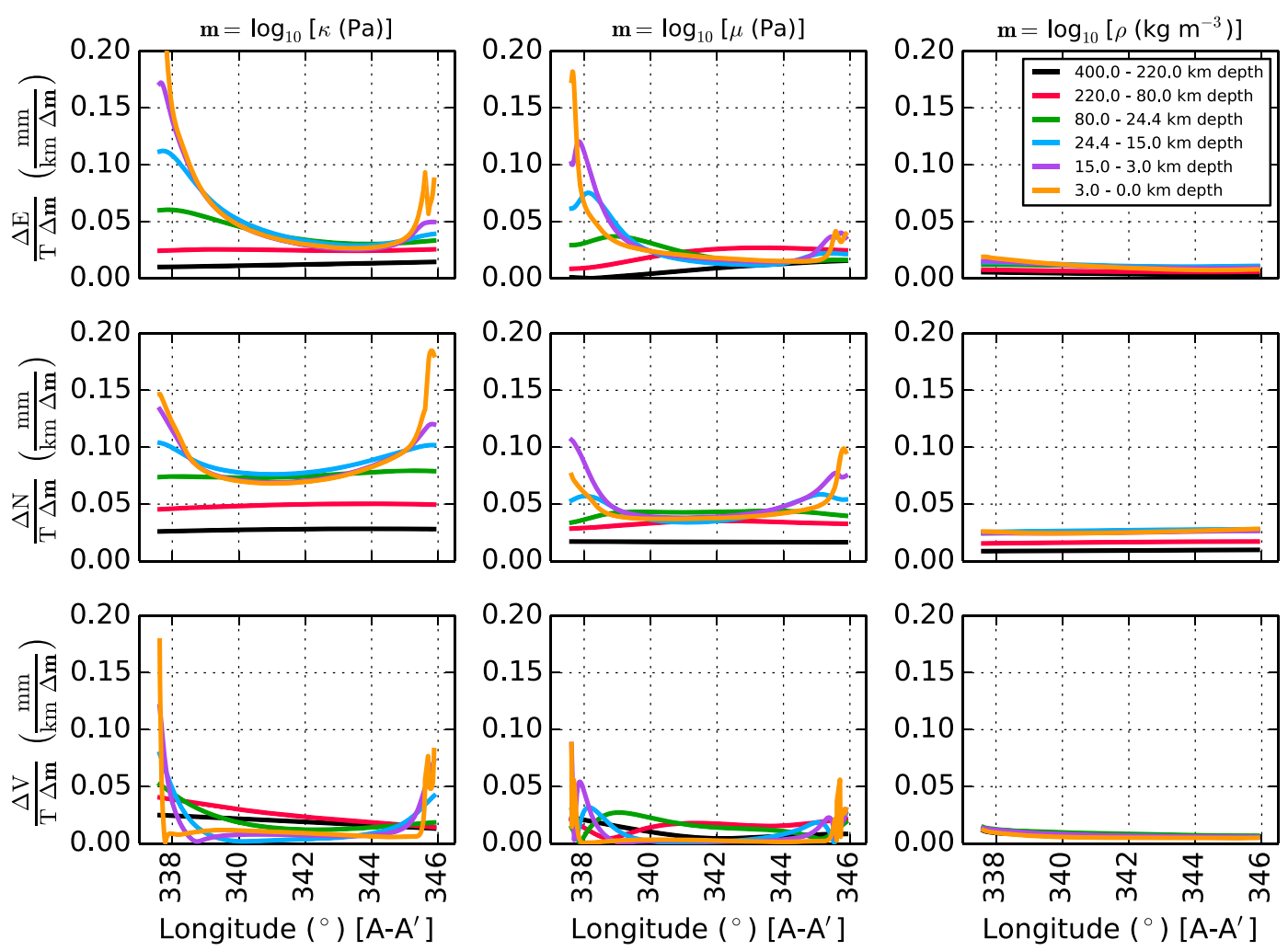

Figure 16. Same as Figure 15 but normalized by the layer thickness, $\mathrm{T}$, in kilometers. The discontinuous jump in the sensitivity just west of $346^{\circ}$, derived from perturbed upper crustal structure (orange line), is caused by the presence of an ocean inlet encountered along the profile.

a function of distance to the coast shifts farther inland for perturbations to deeper structure. In general, perturbations to shallow crustal structure primarily affect the predicted surface displacements at coastal sites and at short wavelengths. In contrast, perturbations to mantle structure mostly affect the predicted surface displacements at longer wavelengths and beyond about $50 \mathrm{~km}$ inland of the coast.

Sensitivities in the vertical component tend to be higher at prediction sites on the southern and western edges of the profiles, where the tidal amplitudes are larger. Moreover, small deviations from a smooth coastline can generate jumps in the sensitivity profiles, such as when an ocean inlet is encountered on the eastern side of the profile line. Analogous to the displacement LGFs, the sensitivity to $1 \%$ perturbations in density structure is generally weaker than the sensitivity to $1 \%$ perturbations in the elastic structure and probably mostly reflects changes in the total Earth mass generated by the perturbation. When scaled by the layer thickness, sensitivities are typically strongest for perturbations to shallow structure, particularly near the coastlines.

It is important to note that the sensitivity kernels we present here are specific to the prediction sites that we have selected in Iceland as well as to the $M_{2}$ ocean tide model. Thus, the sensitivity kernels for the predicted OTL-induced surface displacements across the profiles in Iceland should be considered examples, albeit illustrative and representative of the sensitivities expected for $M_{2}$ OTL in many locations around the globe.

The sensitivity kernels for the OTL-induced surface displacements (e.g., Figure 15) constitute a key element in formulating the inverse problem. In particular, the kernels could be used to relate structural models to displacement observations in a linear inverse problem and to investigate the resolution of the model parameters with application to geodetic tomography. Synthetic testing could reveal better the extent to which perturbations in the elastic moduli and density could be resolved independently for the particular geographic locations considered and the $\mathrm{M}_{2}$ ocean tide model. 


\section{Discussion}

We have explored the theoretical sensitivities of potential, load, and shear Love numbers as well as displacement LGFs to systematic perturbations in elastic Earth structure using both quasi-analytical and numerical techniques. Following the methodology of Okubo and Saito [1983], we developed profiles of LLN partial derivatives for a variety of spherical harmonic degrees (Figures 1-3 and S1-S17). Perturbations to the elastic structure very near the surface strongly affect the high-degree LLNs, as expected due to the short wavelengths of Legendre polynomials at large $n$. The high-degree LLNs are most sensitive to structural perturbations within a skin depth of approximately $(a / n)$ of the surface [Okubo, 1988a], where $a$ is Earth's radius and $n$ is the spherical harmonic degree. The sensitivity of the high-degree LLNs to perturbations in elastic and density structure rapidly approaches zero beyond a few skin depths (Figures 2 and 3). The density kernel constitutes an exception, since a perturbation to density at any depth generates a change in the total Earth mass.

We also computed numerically the sensitivities of displacement LGFs to systematic perturbations in elastic material properties (Figures $6-8$ ). The patterns of sensitivity vary as a function of perturbation depth as well as the angular distance between the measurement site and the load point for each of the three model parameters considered. The distinct patterns exhibited by each of the model parameters leave open the possibility that the two elastic moduli and density may be independently constrained through inversion of observed SML-induced surface displacements.

As a general rule of thumb, illustrated by Figures 6 and 7, the elastic structure at a depth of $D$ km strongly influences the displacement LGFs at a horizontal distance of $D \mathrm{~km}$ from the load point [cf. Okubo, 1988b; Ito and Simons, 2011]. We can also see from Figures 6 and 7 that the sensitivities of SML-induced surface displacements to perturbations in density structure appear to be much weaker than for equivalent perturbations to the two elastic moduli [cf. Baker, 1980].

Furthermore, since the equations of motion account for the gravitational force exerted on the surface mass, increasing the density in any layer will increase the magnitude of the gravitational force exerted at the surface. An increased gravitational force attracting the mass load is consistent with the predominantly negative sensitivity of the displacement LGFs to positive perturbations in density, the approximately constant sensitivity as a function of perturbation depth, and the relatively weak sensitivity observed for perturbations to thin crustal layers (Figures 7 and S19) [cf. Baker, 1980]. By this interpretation, we suggest that changes in the gravitational force, arising from changes to the total Earth mass due to perturbations in density structure, account for the most significant contribution to $K_{\rho}$. Unsurprisingly, the magnitude of LGF sensitivity to density perturbations increases with thickness of the perturbed layer. In contrast, the depth of a perturbation to the elastic moduli plays a significant role in enhancing (shallower) or diminishing (deeper) the LGF sensitivity.

Since combinations of positive and negative perturbations to an array of spherical shells and elastic parameters can theoretically produce equivalent surface displacements at a variety of observer-to-load angular distances, the inverse problem exhibits nonuniqueness, particularly when only a small number of measurement sites are considered [cf. Baker, 1980]. In other words, different Earth models could potentially explain the same observations of SML-induced surface displacements. Moreover, Figures 15 and S21 show that perturbations to thick layers in the mantle can produce larger changes in the OTL-induced surface displacements than perturbations to thin layers in the crust. Perturbations applied to near-surface structure, however, predominantly affect the OTL-induced surface displacements at locations near the load, corresponding to stations along the coast; perturbations to deeper structure generate changes at longer wavelengths and often exhibit peak sensitivities farther inland. The nonuniqueness of the inverse problem may therefore be mitigated with a large and spatially distributed set of SML response observations, which should be explored further through case-specific investigations of model resolution. The sensitivity of load-induced surface displacements to perturbations in structure also depends strongly on the distribution of the global load. Thus, the structural sensitivities of SML-induced surface displacements are load and site specific.

In addition to adequate model resolution, the ability to use observations of SML-induced surface displacements to constrain solid Earth structure also requires the structural sensitivity of the deformation response to exceed observational and modeling errors. In the special case of OTL, peak sensitivities to structure are typically associated with the locations of the largest OTL-induced surface displacements, which generally coincide with coastal sites immediately adjacent to large-amplitude tides offshore. For most geodetic networks, however, only a spatially limited number of stations are deployed near the coast. Furthermore, even 
with a very dense network along the coast, the primary sensitivity would be to near-surface structure. Thus, to improve the ability to detect deeper mantle structure, OTL-induced surface displacements detected farther inland must also be explored. The inland sites, however, tend to exhibit smaller displacement responses as well as weaker structural sensitivities and therefore require more accurate empirical measurements of the OTL-induced deformation as well as minimal errors in the forward model.

Errors affecting the precision and accuracy of the forward model might arise from the numerical derivation of the LGFs, the development and resolution of the ocean tide model, deficiencies in the SNREI Earth model, and the numerical convolution scheme. Errors in the ocean tide model, in particular, tend to be largest near the coast and therefore disproportionately impact measurement sites near the coastline. Uncertainties affecting the observational precision may include contributions from the data acquisition and processing as well as the modeling techniques used to extract the individual tidal harmonics. SEBT displacement response estimates, for example, are often removed at the GPS processing stage and can be erroneous at the $\sim 1 \mathrm{~mm}$ level [e.g., Yuan et al., 2013]. The SEBTs, however, operate primarily at long wavelengths, or global spatial scales, and therefore sample more or less an average of Earth structure [e.g., Latychev et al., 2009]. Thus, for a regional GPS network, any inaccuracies in the SEBT model would likely manifest predominantly as a residual displacement common to the entire network.

Recently, Bos et al. [2015] reported that observations and predictions of OTL-induced surface displacements across western Europe were of sufficient precision to explore structural deficiencies, including both elastic and anelastic deficiencies. In addition, Martens et al. [2016] demonstrated that after the removal of a uniform displacement factor, vector differences between predicted $\mathrm{M}_{2}$ OTL-induced surface displacements generated from a selection of modern ocean tide models coincided with the approximate level of structural sensitivity for reasonable variations in SNREI Earth structure (Figure 12). It should also be recalled that the sensitivity analyses presented here involve differential OTL-induced surface displacements, and therefore, errors related to the particular tide model and convolution scheme, which do not change between forward model computations, cancel out to within about a fraction of a micron.

We also reiterate that the sensitivity kernels presented here were derived for SNREI Earth models. At this stage, we have not considered the effects of anelasticity, anisotropy, or lateral heterogeneities on the sensitivities of LLNs, LGFs, and OTL-induced surface displacements to perturbations in structure. Bos et al. [2015] found that dissipation effects within the asthenosphere could account for up to about $0.3 \mathrm{~mm}$ of residual OTL-induced surface displacements in western Europe and that allowing for anisotropy could reduce the mean residual by approximately $0.1 \mathrm{~mm}$. Incorporating an anelastic constitutive relation into the equations of motion produces complex-valued LLNs; thus, the differences between observed and predicted OTL-induced surface displacements would also include a small phase delay.

Regarding lateral heterogeneities, the computations of the LLNs and LGFs require radially symmetric structure by design. To explore the effects of lateral heterogeneities on the predicted SML-induced surface displacements, one might compute discrete grids of local LGFs [e.g., Dill et al., 2015]. The Earth's displacement response to SML, however, depends on global Earth structure and particularly on the material properties spanning the region between the load and the observer. Thus, grids of local LGFs would not be particularly useful for regional or global studies, where stations might be located at a variety of distances from spatially complicated loads, as in the case of OTL. A better technique would be to use fully numerical approaches, such as finite element or spectral element methods. Using numerical techniques, albeit applied to the SEBTs rather than SML, Latychev et al. [2009] showed that realistic three-dimensional variations in structure can perturb radial displacements by an amount on the order of $1 \mathrm{~mm}$ in the semidiurnal tidal band.

It is also worth recalling that the forward model generates predicted SML-induced surface displacements based on a model for Earth structure as well as a load model. Here we have focused on the sensitivity of OTL-induced surface displacements to perturbations in elastic structure and taken the load model as given. In some cases, however, the Earth model could be considered given and the observations of SML-induced surface displacements used to constrain the spatial extent and volume of a load. 


\section{Summary and Conclusions}

We have computed the sensitivities of Love numbers (potential, load, and shear), displacement load Green's functions, and $M_{2}$ OTL-induced surface displacements to perturbations in elastic and density structure through the crust and mantle. In each case, the sensitivities depend on the depth of the structural perturbation, the thickness of the perturbed layer, and the particular parameter that was perturbed (e.g., $\kappa, \mu$, and $\rho$ ). The sensitivities of the Love numbers additionally depend on the spherical harmonic degree of the deformation, since different degrees sample structure at different wavelengths. The sensitivities of the LGFs further depend on the angular separation between the measurement site and the location of the applied (point source) mass load. Furthermore, the sensitivities of the OTL-induced surface displacements are influenced by the tide model as well as the location of the measurement site.

Although the LLNs, LGFs, and OTL-induced surface displacements are sensitive to perturbations in both elastic and density structure, the sensitivity of the deformation response to perturbations in density structure appears to be relatively weak unless applied to a very thick layer (Figures 6 and 7). We attribute the patterns of density sensitivity primarily to increases in the total Earth mass that occur with perturbations to the density and that generate deviations in the surface gravity. We find that most of the sensitivity to perturbations in the elastic moduli is concentrated within $500 \mathrm{~km}$ depth of the surface and within $10^{\circ}$ of the load point (i.e., within an angular distance of $10^{\circ}$ between the load and the observer).

Different combinations of positive and negative perturbations to the elastic and density structure of the crust and upper mantle can theoretically generate equivalent OTL-induced surface displacements at measurement sites on the surface, implying a nonuniqueness of the inverse problem. The apparent nonuniqueness might be mitigated through appropriately dense and strategically distributed geodetic networks, since structural perturbations applied at different depths influence the surface deformation more strongly at different distances from the load point (Figures 7, 15, and 16). Moreover, the spatially and temporally complicated patterns of ocean tidal loads can further facilitate the sampling of structure at a variety of wavelengths.

In conclusion, the possibility of performing geodetic tomography using observations of Earth deformation induced by surface mass loading depends on many factors, including the specific geodetic network, the quality of the geodetic data, the computational methods, the quality and spatial distribution of the load model, and the sensitivity to structure. The sensitivity kernels presented here, however, lay the foundation for future tomographic studies since, along with models for the observational and prediction error, the kernels could be used to formulate the inverse problem.

We thank Duncan Agnew and Richard Ray for their fruitful discussions on ocean tidal loading as well as thoughtful suggestions regarding the development of our loading response convolution code. We also thank

Shuhei Okubo for valuable guidance in understanding the development of the Love number variational equations. We gratefully acknowledge the associate editor and two anonymous reviewers for their insightful and comprehensive comments, which substantially strengthened the manuscript. We also acknowledge support from the National Science Foundation Geophysics Program funding under grant EAR-1417245. This manuscript is based upon work supported by the NASA Earth and Space Science Fellowship to HRM under grant NNX14AO04H. Some figures were generated using Generic Mapping Tools [Wessel et al., 2013]. The load Love numbers and displacement load Green's functions computed for this study are provided in the supporting information (Data Sets S7-S24). The reference Earth models used to derive the Love numbers and Green's functions are also provided in the supporting information (Data Sets S1-S6).

\section{References}

Abramowitz, M., and I. A. Stegun (1964), Handbook of Mathematical Functions: With Formulas, Graphs, and Mathematical Tables, Courier, Dover, New York.

Agnew, D. C. (1997), NLOADF: A program for computing ocean-tide loading, J. Geophys. Res., 102(B3), 5109-5110.

Agnew, D. C. (2012), SPOTL: Some programs for ocean-tide loading, Scripps Inst. of Oceanogr. Tech. Rep., Scripps Inst. of Oceanogr.,

La Jolla, Calif.

Agnew, D. C. (2015), Earth tides, in Treatise on Geophysics, vol. 3, edited by T. Herring and G. Schubert, pp. 151-178, Elsevier, Amsterdam. Alterman, Z., H. Jarosch, and C. Pekeris (1959), Oscillations of the Earth, Proc. R. Soc. A, 252(1268), 80-95.

Amante, C., and B. W. Eakins (2009), ETOPO1 1 Arc-Minute Global Relief Model: Procedures, Data Sources and Analysis, U.S. Dep. of Commer., Boulder, Colo.

Baker, T. (1980), Tidal tilt at Llanrwst, North Wales: Tidal loading and Earth structure, Geophys. J. Int., 62(2), 269-290,

doi:10.1111/j.1365-246X.1980.tb04855.x.

Baker, T. (1984), Tidal deformations of the Earth, Sci. Prog. Oxford, 69, 197-233.

Baker, T. F., and M. S. Bos (2003), Validating Earth and ocean tide models using tidal gravity measurements, Geophys. J. Int., 152(2), 468-485, doi:10.1046/j.1365-246X.2003.01863.x.

Blewitt, G. (2003), Self-consistency in reference frames, geocenter definition, and surface loading of the solid Earth, J. Geophys. Res., 108(B2), 2103, doi:10.1029/2002JB002082.

Bos, M. (2010), Comment on "Anomalous ocean load tide signal observed in lake-level variations in Tierra del Fuego" by A. Richter et al., Geophys. Res. Lett., 37, L04303, doi:10.1029/2009GL041531.

Bos, M., and T. Baker (2005), An estimate of the errors in gravity ocean tide loading computations, J. Geod., 79(1), 50-63.

Bos, M. S., N. T. Penna, T. F. Baker, and P. J. Clarke (2015), Ocean tide loading displacements in western Europe. Part 2: GPS-observed anelastic dispersion in the asthenosphere, J. Geophys. Res. Solid Earth, 120, 6540-6557, doi:10.1002/2015JB011884.

Chu, R., B. Schmandt, and D. V. Helmberger (2012), Upper mantle $P$ velocity structure beneath the Midwestern United States derived from triplicated waveforms, Geochem. Geophys. Geosyst., 13, Q0AK04, doi:10.1029/2011GC003818.

Dill, R., V. Klemann, Z. Martinec, and M. Tesauro (2015), Applying local Green's functions to study the influence of the crustal structure on hydrological loading displacements, J. Geodyn., 88, 14-22.

Dziewonski, A. M., and D. L. Anderson (1981), Preliminary reference Earth model, Phys. Earth Planet. Inter., 25(4), 297-356, doi:10.1016/0031-9201(81)90046-7.

Egbert, G. D., and S. Y. Erofeeva (2002), Efficient inverse modeling of barotropic ocean tides, J. Atmos. Oceanic Technol., 19 (2), $183-204$. 
Egbert, G. D., S. Y. Erofeeva, and R. D. Ray (2010), Assimilation of altimetry data for nonlinear shallow-water tides: Quarter-diurnal tides of the Northwest European Shelf, Cont. Shelf Res., 30(6), 668-679, doi:10.1016/j.csr.2009.10.011.

Farrell, W. (1972), Deformation of the Earth by surface loads, Rev. Geophys., 10(3), 761-797.

Farrell, W. (1973), Earth tides, ocean tides and tidal loading, Philos. Trans. R. Soc. A, 274(1239), 253-259, doi:10.1098/rsta.1973.0050.

Francis, O., and P. Mazzega (1990), Global charts of ocean tide loading effects, J. Geophys. Res., 95(C7), 11,411-11,424, doi:10.1029/JC095iC07p11411.

Fu, Y., J. T. Freymueller, and T. van Dam (2012), The effect of using inconsistent ocean tidal loading models on GPS coordinate solutions, J. Geod., 86(6), 409-421.

Gilbert, F., and A. Dziewonski (1975), An application of normal mode theory to the retrieval of structural parameters and source mechanisms from seismic spectra, Philos. Trans. R. Soc. A, 278(1280), 187-269.

Goad, C. C. (1980), Gravimetric tidal loading computed from integrated Green's functions, J. Geophys. Res., 85(B5), 2679-2683, doi:10.1029/JB085iB05p02679.

Grand, S. P., and D. V. Helmberger (1984), Upper mantle shear structure of North America, Geophys. J. Int., 76(2), 399-438, doi:10.1111/j.1365-246X.1984.tb05053.x.

Guo, J., Y. Li, Y. Huang, H. Deng, S. Xu, and J. Ning (2004), Green's function of the deformation of the Earth as a result of atmospheric loading, Geophys. J. Int., 159(1), 53-68, doi:10.1111/j.1365-246X.2004.02410.x.

Harrison, J. (1985), Earth Tides (Benchmark Papers in Geology), Van Nostrand Reinhold, New York.

Ito, T., and M. Simons (2011), Probing asthenospheric density, temperature, and elastic moduli below the western United States, Science, 332(6032), 947-951, doi:10.1126/science.1202584.

Jeffreys, H. (1961), Small corrections in the theory of surface waves, Geophys. J. Int., 6(1), 115-117, doi:10.1111/j.1365-246X.1961.tb02965.x. Jordan, T. H. (1978), Composition and development of the continental tectosphere, Nature, 274, 544-548.

Kennett, B., E. Engdahl, and R. Buland (1995), Constraints on seismic velocities in the Earth from traveltimes, Geophys. J. Int., 122(1), 108-124, doi:10.1111/j.1365-246X.1995.tb03540.x.

Kustowski, B., G. Ekström, and A. Dziewoński (2008), Anisotropic shear-wave velocity structure of the Earth's mantle: A global model, J. Geophys. Res., 113, B06306, doi:10.1029/2007JB005169.

Lambeck, K. (1988), Geophysical Geodesy: The Slow Deformations of the Earth, Clarendon Press, Oxford, U. K.

Lanzano, P. (1982), Deformations of an Elastic Earth, Int. Geophys. Ser., vol. 31, Academic Press, New York.

Latychev, K., J. X. Mitrovica, M. Ishii, N.-H. Chan, and J. L. Davis (2009), Body tides on a 3-D elastic Earth: Toward a tidal tomography, Earth Planet. Sci. Lett., 277(1-2), 86-90, doi:10.1016/j.epsl.2008.10.008.

Longman, I. (1962), A Green's function for determining the deformation of the Earth under surface mass loads: 1. Theory, J. Geophys. Res., 67(2), 845-850, doi:10.1029/JZ067i002p00845.

Longman, I. (1963), A Green's function for determining the deformation of the Earth under surface mass loads: 2. Computations and numerical results, J. Geophys. Res., 68(2), 485-496, doi:10.1029/JZ068i002p00485.

Love, A. E. H. (1911), Some Problems of Geodynamics: Being an Essay to Which the Adams Prize in the University of Cambridge Was Adjudged in 1911, Cambridge Univ. Press, Cambridge, U. K.

Martens, H. R., M. Simons, S. Owen, and L. Rivera (2016), Observations of ocean tidal load response in South America from subdaily GPS positions, Geophys. J. Int., 205, 1637-1664, doi:10.1093/gji/ggw087.

Melchior, P. (1983), The Tides of the Planet Earth, 2nd ed., p. 653, Pergamon Press, Oxford, U. K.

Molodenskiy, S. (1977), Relation between Love numbers and load factors, Izv. Earth Phys., 13(3), 147-149.

Montagner, J.-P., and B. Kennett (1996), How to reconcile body-wave and normal-mode reference Earth models, Geophys. J. Int., 125(1), 229-248, doi:10.1111/j.1365-246X.1996.tb06548.x.

Munk, W., and G. MacDonald (1960), The Rotation of the Earth, p. 323, Cambridge Univ. Press, New York.

$\mathrm{Na}$, S.-H., and J. Baek (2011), Computation of the load Love number and the load Green's function for an elastic and spherically symmetric Earth, J. Korean Phys. Soc., 58(5), 1195-1205.

Okubo, S. (1988a), Asymptotic solutions to the static deformation of the Earth-I. Spheroidal mode, Geophys. J. Int., 92(1), 39-51, doi:10.1111/j.1365-246X.1988.tb01119.x.

Okubo, S. (1988b), Green's function to a point load: Its dependence on Earth model, Bull. Inf. Bur. Gravimetrique Int., 62, $68-74$.

Okubo, S., and T. Endo (1986), Static spheroidal deformation of degree 1-Consistency relation, stress solution and partials, Geophys. J. Int., 86(1), 91 -102, doi:10.1111/j.1365-246X.1986.tb01074.x.

Okubo, S., and M. Saito (1983), Partial derivative of Love numbers, Bull. Geodesique, 57(1), 167-179.

Penna, N. T., M. S. Bos, T. F. Baker, and H.-G. Scherneck (2008), Assessing the accuracy of predicted ocean tide loading displacement values, J. Geod., 82(12), 893-907, doi:10.1007/s00190-008-0220-2.

Penna, N. T., P. J. Clarke, M. S. Bos, and T. F. Baker (2015), Ocean tide loading displacements in western Europe. Part 1: Validation of kinematic GPS estimates, J. Geophys. Res. Solid Earth, 120, 6523-6539, doi:10.1002/2015JB011882.

Press, W. H., S. A. Teukolsky, W. T. Vetterling, and B. P. Flannery (2007), Numerical Recipes 3rd Edition: The Art of Scientific Computing, Cambridge Univ. Press, Cambridge, U. K.

Saito, M. (1978), Relationship between tidal and load Love numbers, J. Phys. Earth, 26(1), 13-16.

Stammer, D., et al. (2014), Accuracy assessment of global barotropic ocean tide models, Rev. Geophys., 52, 243-282, doi:10.1002/2014RG000450.

Takeuchi, H. (1950), On the Earth tide of the compressible Earth of variable density and elasticity, Eos Trans. AGU, 31(5), 651-689, doi:10.1029/TR031i005p00651.

Takeuchi, H., and M. Saito (1972), Seismic surface waves, in Methods in Computational Physics: Seismology: Surface Waves and Earth Oscillations, vol. 11, edited by B. A. Bolt et al., pp. 217-295, Academic Press, New York.

Tarantola, A. (2005), Inverse Problem Theory And Methods for Model Parameter Estimation, Soc. for Ind. and Appl. Math, Philadelphia, Pa.

Wang, H., L. Xiang, L. Jia, L. Jiang, Z. Wang, B. Hu, and P. Gao (2012), Load Love numbers and Green's functions for elastic Earth models PREM, iasp91, ak135, and modified models with refined crustal structure from Crust 2.0, Comput. Geosci., 49, 190-199, doi:10.1016/j.cageo.2012.06.022.

Wessel, P., and W. H. Smith (1996), A global, self-consistent, hierarchical, high-resolution shoreline database, J. Geophys. Res., 101(B4), $8741-8743$, doi:10.1029/96JB00104.

Wessel, P., W. H. Smith, R. Scharroo, J. Luis, and F. Wobbe (2013), Generic Mapping Tools: Improved version released, Eos Trans. AGU, 94(45), 409-410.

Wiggins, R. A. (1968), Terrestrial variational tables for the periods and attenuation of the free oscillations, Phys. Earth Planet. Inter., 1(4), 201-266, doi:10.1016/0031-9201(68)90062-9. 
Wu, X., J. Ray, and T. van Dam (2012), Geocenter motion and its geodetic and geophysical implications, J. Geodyn., 58, 44-61.

Yuan, L., and B. F. Chao (2012), Analysis of tidal signals in surface displacement measured by a dense continuous GPS array, Earth Planet. Sci. Lett., 355, 255-261, doi:10.1016/j.epsl.2012.08.035.

Yuan, L., B. F. Chao, X. Ding, and P. Zhong (2013), The tidal displacement field at Earth's surface determined using global GPS observations, J. Geophys. Res. Solid Earth, 118, 2618-2632, doi:10.1002/jgrb.50159. 